\title{
DERIVED EQUIVALENCES AND STABLE EQUIVALENCES OF MORITA TYPE, I
}

\author{
WEI HU AND CHANGCHANG XI
}

\section{Dedicated to Professor Shaoxue Liu on the occasion of his 80 th birthday}

\begin{abstract}
For self-injective algebras, Rickard proved that each derived equivalence induces a stable equivalence of Morita type. For general algebras, it is unknown when a derived equivalence implies a stable equivalence of Morita type. In this article, we first show that each derived equivalence $F$ between the derived categories of Artin algebras $A$ and $B$ arises naturally as a functor $\bar{F}$ between their stable module categories, which can be used to compare certain homological dimensions of $A$ with that of $B$. We then give a sufficient condition for the functor $\bar{F}$ to be an equivalence. Moreover, if we work with finite-dimensional algebras over a field, then the sufficient condition guarantees the existence of a stable equivalence of Morita type. In this way, we extend the classical result of Rickard. Furthermore, we provide several inductive methods for constructing those derived equivalences that induce stable equivalences of Morita type. It turns out that we may produce a lot of (usually not self-injective) finite-dimensional algebras that are both derived-equivalent and stably equivalent of Morita type; thus, they share many common invariants.
\end{abstract}

\section{$\S 1$. Introduction}

As is well known, derived equivalence and stable equivalence of Morita type are two fundamental types of equivalences in algebras. Roughly, the former is the equivalence of the derived categories of their module categories, while the latter is the equivalence of their module categories modulo projective direct summands, where the equivalence is given by tensoring a

Received November 17, 2009. Revised February 15, 2010. Accepted February 24, 2010.

2000 Mathematics Subject Classification. Primary 18E30, 16G10; Secondary 18G20, $16 \mathrm{D} 90$.

Xi's work partially supported by the National Natural Science Foundation of China grant (10731070).

(C) 2011 by The Editorial Board of the Nagoya Mathematical Journal 
two-sided module (see Section 2 for more detail). They play an important role in the modern representation theory of groups and algebras, transfer information from one algebra to another, and provide a convenient bridge between two different (derived or stable) categories. In particular, derived equivalences preserve many significant invariants, for example, the center of an algebra, the number of nonisomorphic simple modules, the Hochschild cohomology groups, and Cartan determinants. Stable equivalences of Morita type, introduced in around 1990 (see, e.g., [3]) and appearing frequently in the block theory of finite groups, preserve also many nice invariants, for instance, the global, finitistic, and representation dimensions (see [19]) as well as the representation types (see [8]). For self-injective algebras, the two notions are closely related to each other; this was revealed by a well-known result of Rickard [15], which states that a derived equivalence between selfinjective algebras always induces a stable equivalence of Morita type. Moreover, the remarkable abelian defect group conjecture of Broué, which states that the module categories of a block algebra $A$ of a finite group algebra and its Brauer correspondent $B$ should have equivalent derived categories if their common defect group is abelian (see [16]), makes the two concepts more attractive and intimate. For general finite-dimensional algebras, however, derived equivalence and stable equivalence of Morita type seem to be completely different from each other; for example, a representation-finite algebra may be derived-equivalent to a representation-infinite algebra via a tilting module, and consequently, they neither are stably equivalent of Morita type nor have the same representation dimension. Thus, a natural question arises: what kind of relationship between a derived equivalence and a stable equivalence of Morita type for general finite-dimensional algebras could exist? In other words, we consider the following question.

Question. When does a derived equivalence between two finitedimensional (not necessarily self-injective) algebras $A$ and $B$ induce a stable equivalence of Morita type between them?

Thus, a positive answer to the above question would let us know more invariants between the algebras $A$ and $B$. However, to date, little is known about this question. One does not even know when a derived equivalence induces a stable equivalence for general finite-dimensional algebras.

In this article, we provide some answers to this question. To state our main result, let us introduce the notion of an almost $\nu$-stable functor. Suppose that $F$ is a derived equivalence between two Artin algebras $A$ and $B$, 
with a quasi-inverse functor $G$. Further, suppose that

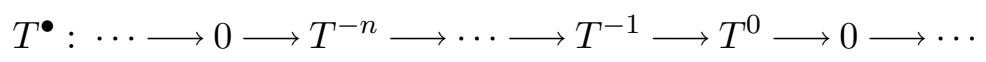

is a radical tilting complex over $A$ associated to $F$, and suppose that

$$
\bar{T}^{\bullet}: \cdots \longrightarrow 0 \longrightarrow \bar{T}^{0} \longrightarrow \bar{T}^{1} \longrightarrow \cdots \longrightarrow \bar{T}^{n} \longrightarrow 0 \longrightarrow \cdots
$$

is a radical tilting complex over $B$ associated to $G$. The functor $F$ is called almost $\nu$-stable if $\operatorname{add}\left(\bigoplus_{i=-1}^{-n} T^{i}\right)=\operatorname{add}\left(\bigoplus_{i=-1}^{-n} \nu_{A} T^{i}\right)$, and $\operatorname{add}\left(\bigoplus_{i=1}^{n} \bar{T}^{i}\right)=$ $\operatorname{add}\left(\bigoplus_{i=1}^{n} \nu_{B} \bar{T}^{i}\right)$, where $\nu_{A}$ is the Nakayama functor of $A$. Note that the summations exclude only the term in degree 0. If $A$ and $B$ are self-injective, then every derived equivalence between $A$ and $B$ is almost $\nu$-stable (by Proposition 3.8 below). Surprisingly, even beyond the class of self-injective algebras, there are plenty of almost $\nu$-stable derived equivalences, for example, the derived equivalences constructed in [6, Corollary 3.8] and in Proposition 3.11 below. In fact, we give a general machinery below to produce such derived equivalences.

With this notion in mind, our main result can be stated as follows.

TheOREM 1.1. Let $A$ and $B$ be Artin algebras, and let $F$ be a derived equivalence between $A$ and $B$. Then,

(1) $F$ induces a functor $\bar{F}$ from the stable module category over $A$ to that over $B$;

(2) if $F$ is almost $\nu$-stable, then the functor $\bar{F}$ defined in (1) is an equivalence, and further, if $F$ is an almost $\nu$-stable derived equivalence between finite-dimensional algebras $A$ and $B$ over a field $k$, then there is a stable equivalence $\Phi$ of Morita type between $A$ and $B$ such that $\Phi(X) \simeq \bar{F}(X)$ for all objects $X$ in the stable module category over $A$.

As a consequence of the proof of Theorem 1.1, we have the following facts on homological dimensions of algebras.

Corollary 1.2. Let $A$ and $B$ be Artin algebras, and let $F$ be a derived equivalence between $A$ and $B$. If $\operatorname{add}\left(\bigoplus_{i=-1}^{-n} T^{i}\right)=\operatorname{add}\left(\bigoplus_{i=-1}^{-n} \nu_{A} T^{i}\right)$, then

(1) $\operatorname{gl} \cdot \operatorname{dim}(A) \leq$ gl.dim $(B)$;

(2) $\operatorname{fin} \cdot \operatorname{dim}(A) \leq$ fin.dim $(B)$;

(3) dom.dim $(A) \geq \operatorname{dom} \cdot \operatorname{dim}(B)$;

where $\operatorname{gl} \cdot \operatorname{dim}(A)$, fin.dim $(A)$, and $\operatorname{dom} \cdot \operatorname{dim}(A)$ stand for the global, finitistic, and dominant dimensions of $A$, respectively. 
Note that if $A$ and $B$ are finite-dimensional self-injective, we reobtain the well-known result of Rickard [15] from Theorem 1.1: derived-equivalent self-injective algebras are stably equivalent of Morita type. Moreover, Theorem 1.1 allows us to obtain a lot of (usually not self-injective) algebras which are both derived-equivalent and stably equivalent of Morita type. By the corollary below, we can even repeatedly construct derived equivalences satisfying the almost $\nu$-stable condition.

Corollary 1.3. Let $k$ be a field, and let $F$ be an almost $\nu$-stable derived equivalence between two finite-dimensional $k$-algebras $A$ and $B$. Then,

(1) for any finite-dimensional self-injective $k$-algebra $C$, there is an almost $\nu$-stable derived equivalence between the two tensor products $A \otimes_{k} C$ and $B \otimes_{k} C$;

(2) let $\bar{F}$ be the stable equivalence induced by $F$ in Theorem 1.1; then, for each $A$-module $X$, there is an almost $\nu$-stable derived equivalence between the endomorphism algebras $\operatorname{End}_{A}(A \oplus X)$ and $\operatorname{End}_{B}(B \oplus \bar{F}(X))$;

(3) if $X$ is an $A$-module such that $F(X)$ is isomorphic to a $B$-module $Y$, then there is an almost $\nu$-stable derived equivalence between the one-point extensions $A[X]$ and $B[Y]$.

This article is organized as follows. In Section 2, we recall some basic definitions and facts required in proofs. In Section 3, we first show that every derived equivalence $F$ between two Artin algebras $A$ and $B$ gives rise to a functor $\bar{F}$ between their stable module categories, and we then give a sufficient condition for the functor $\bar{F}$ to be an equivalence. In Section 4 , we deduce some properties of the functor $\bar{F}$ and then compare homological dimensions of $A$ with that of $B$. In particular, we get Corollary 1.2. As a by-product, we reobtain a result in [12] that a derived equivalence preserves the finiteness of finitistic dimension. In Section 5, we show that the condition given in Section 3 is sufficient for $F$ to induce a stable equivalence of Morita type when we work with finite-dimensional algebras over a field. In Section 6, we give several methods to construct inductively derived equivalences satisfying the almost $\nu$-stable condition. Finally, in Section 7 , we exhibit a couple of examples to explain our points about the main result.

\section{$\S 2 . \quad$ Preliminaries}

In this section, we recall basic definitions and facts required in our proofs.

Let $\mathcal{C}$ be an additive category. 
Convention. For two morphisms $f: X \rightarrow Y$ and $g: Y \rightarrow Z$ in $\mathcal{C}$, the composition of $f$ with $g$ is written as $f g$, which is a morphism from $X$ to $Z$. But for two functors $F: \mathcal{C} \rightarrow \mathcal{D}$ and $G: \mathcal{D} \rightarrow \mathcal{E}$ of categories, their composition is denoted by $G F$.

For an object $X$ in $\mathcal{C}$, we denote by $\operatorname{add}(X)$ the full subcategory of $\mathcal{C}$ consisting of all direct summands of finite direct sums of copies of $X$.

Throughout this article, unless specified otherwise, all algebras will be Artin algebras over a fixed commutative Artin ring $R$. All modules will be finitely generated unitary left modules. If $A$ is an Artin algebra, the category of all modules over $A$ is denoted by $A$-mod; the full subcategory of $A$-mod consisting of projective (resp., injective) modules is denoted by $A$-proj (resp., $A$-inj). We denote by $D$ the usual duality on $A$-mod. The duality $\operatorname{Hom}_{A}(-, A)$ from $A$-proj to $A^{\text {op }}$-proj is denoted by ${ }^{*}$; that is, for each projective $A$-module $P$, the projective $A^{\text {op }}$-module $\operatorname{Hom}_{A}(P, A)$ is denoted by $P^{*}$. We denote by $\nu_{A}$ the Nakayama functor $D \operatorname{Hom}_{A}(-, A): A$-proj $\longrightarrow$ $A$-inj.

The stable module category $A$-mod of an algebra $A$ is, by definition, an $R$-category in which objects are the same as the objects of $A$-mod and, for two objects $X, Y$ in $A$-mod, their morphism set, denoted by $\underline{\operatorname{Hom}}_{A}(X, Y)$, is the quotient of $\operatorname{Hom}_{A}(X, Y)$ modulo the homomorphisms that factorize through projective modules. Two algebras are said to be stably equivalent if their stable module categories are equivalent as $R$-categories.

For finite-dimensional algebras over a field $k$, there is a special class of stable equivalences, namely, stable equivalences of Morita type. Recall that two finite-dimensional algebras $A$ and $B$ over a field $k$ are called stably equivalent of Morita type if there are two bimodules ${ }_{A} M_{B}$ and ${ }_{B} N_{A}$ satisfying the following properties:

(1) all of the one-sided modules ${ }_{A} M, M_{B},{ }_{B} N$, and $N_{A}$ are projective, and

(2) there is an $A$-A-bimodule isomorphism ${ }_{A} M \otimes_{B} N_{A} \simeq A \oplus U$ for a projective $A$-A-bimodule $U$, and there is a $B$ - $B$-bimodule isomorphism ${ }_{B} N \otimes_{A} M_{B} \simeq B \oplus V$ for a projective $B$ - $B$-bimodule $V$.

If two finite-dimensional algebras $A$ and $B$ over a field are stably equivalent of Morita type, then the functor $T_{N}: A$-mod $\longrightarrow B$ - $\underline{\bmod }$ defined by ${ }_{B} N \otimes_{A}-$ is an equivalence and is also called a stable equivalence of Morita type. (Note that if we extend the definition of a stable equivalence of Morita type from finite-dimensional algebras to $R$-projective Artin $R$-algebras, then 
there is an open problem of whether $T_{N}$ could induce a stable equivalence on stable module categories, namely, whether ${ }_{A} U \otimes_{A} X$ is projective for every $A$-module $X$.)

Now we recall some definitions relevant to derived equivalences.

Let $\mathcal{C}$ be an additive category. A complex $X^{\bullet}$ over $\mathcal{C}$ is a sequence of morphisms $d_{X}^{i}$ between objects $X^{i}$ in $\mathcal{C}: \cdots \rightarrow X^{i-1} \stackrel{d_{X}^{i-1}}{\longrightarrow} X^{i} \stackrel{d_{X}^{i}}{\longrightarrow} X^{i+1} \stackrel{d_{X}^{i+1}}{\longrightarrow}$ $X^{i+2} \rightarrow \cdots$ such that $d_{X}^{i} d_{X}^{i+1}=0$ for all $i \in \mathbb{Z}$. We write $X^{\bullet}=\left(X^{i}, d_{X}^{i}\right)$. For each complex $X^{\bullet}$, the brutal truncation $\sigma_{<i} X^{\bullet}$ is a quotient complex of $X^{\bullet}$ such that $\left(\sigma_{<i} X^{\bullet}\right)^{k}$ is $X^{k}$ for all $k<i$ and zero otherwise. We define $\sigma_{\geq i} X^{\bullet}$ similarly. The category of complexes over $\mathcal{C}$ is denoted by $\mathscr{C}(C)$. The homotopy category of complexes over $\mathcal{C}$ is denoted by $\mathscr{K}(\mathcal{C})$. When $\mathcal{C}$ is an abelian category, the derived category of complexes over $\mathcal{C}$ is denoted by $\mathscr{D}(\mathcal{C})$. The full subcategory of $\mathscr{K}(\mathcal{C})$ and $\mathscr{D}(\mathcal{C})$ consisting of bounded complexes over $\mathcal{C}$ is denoted by $\mathscr{K}^{\mathrm{b}}(\mathcal{C})$ and $\mathscr{D}^{\mathrm{b}}(\mathcal{C})$, respectively. Moreover, we denote by $\mathscr{C}^{-}(\mathcal{C})$ the category of complexes bounded above, and by $\mathscr{K}^{-}(\mathcal{C})$ the homotopy category of $\mathscr{C}^{-}(\mathcal{C})$. Similarly, we have the category $\mathscr{C}^{+}(\mathcal{C})$ of complexes bounded below and the homotopy category $\mathscr{K}^{+}(\mathcal{C})$ of $\mathscr{C}^{+}(\mathcal{C})$. As usual, for a given Artin algebra $A$, we simply write $\mathscr{C}(A)$ for $\mathscr{C}(A$-mod $), \mathscr{K}(A)$ for $\mathscr{K}(A$-mod $)$, and $\mathscr{K}^{\mathrm{b}}(A)$ for $\mathscr{K}^{\mathrm{b}}(A$-mod $)$. Similarly, we write $\mathscr{D}(A)$ and $\mathscr{D}^{\mathrm{b}}(A)$ for $\mathscr{D}(A$-mod $)$ and $\mathscr{D}^{\mathrm{b}}(A$-mod $)$, respectively.

It is well known that, for an Artin algebra $A$, the categories $\mathscr{K}(A)$ and $\mathscr{D}(A)$ are triangulated categories. For basic results on triangulated categories, we refer to Happel [5]. Throughout this article, we denote by $X[n]$ rather than $T^{n} X$ the object obtained from $X$ by shifting $n$ times. In particular, for a complex $X^{\bullet}$ in $\mathscr{K}(A)$ or $\mathscr{D}(A)$, the complex $X^{\bullet}[1]$ is obtained from $X^{\bullet}$ by shifting $X^{\bullet}$ to the left by one degree.

Let $A$ be an Artin algebra. A homomorphism $f: X \longrightarrow Y$ of $A$-modules is called a radical map if, for any $A$-module $Z$ and homomorphisms $h: Z \longrightarrow$ $X$ and $g: Y \longrightarrow Z$, the composition $h f g$ is not an isomorphism. A complex over $A$-mod is called a radical complex if all of its differential maps are radical. We have the following basic properties of radical complexes.

(a) Every complex over $A$-mod is isomorphic in the homotopy category $\mathscr{K}(A)$ to a radical complex. Indeed, let $X^{\bullet}=\left(X^{i}, d_{X}^{i}\right)$ be a complex in $\mathscr{K}(A)$, and let $d_{X}^{0}$ be not radical. Then there is an indecomposable $A$-module $Y$ and maps $f: Y \longrightarrow X^{0}$ and $g: X^{1} \longrightarrow Y$ such that $f d_{X}^{0} g=1_{Y}$. Let $Y^{\bullet}$ be the complex $\cdots \rightarrow 0 \rightarrow Y \stackrel{1_{Y}}{\longrightarrow} Y \rightarrow 0 \rightarrow \cdots$ with the left $Y$ in degree 0 . We can define a chain map $s^{\bullet}: Y^{\bullet} \longrightarrow X^{\bullet}$ with $s^{0}=f, s^{1}=f d_{X}^{0}$, 
and $s^{i}=0$ for all $i \neq 0,1$. Let $Z^{\bullet}$ be a cokernel of $s^{\bullet}$ in $\mathscr{C}(A)$. Then we have an exact sequence of complexes: $0 \longrightarrow Y^{\bullet} \longrightarrow X^{\bullet} \longrightarrow Z^{\bullet} \longrightarrow 0$ in $\mathscr{C}(A)$. Moreover, for each integer $i$, the exact sequence $0 \longrightarrow Y^{i} \longrightarrow X^{i} \longrightarrow Z^{i} \longrightarrow 0$ is split-exact. By [5, Chapter I, Sections 2 and 3.2], we have a distinguished triangle $Y^{\bullet} \longrightarrow X^{\bullet} \longrightarrow Z^{\bullet} \longrightarrow Y^{\bullet}[1]$ in $\mathscr{K}(A)$. Since $Y^{\bullet} \simeq 0$ in $\mathscr{K}(A)$, we see that $X^{\bullet} \simeq Z^{\bullet}$ in $\mathscr{K}(A)$. In this way, we can reduce every nonradical differential map of a complex to a radical map in $\mathscr{K}(A)$.

(b) If two radical complexes $X^{\bullet}$ and $Y^{\bullet}$ are isomorphic in $\mathscr{K}(A)$, then $X^{\bullet}$ and $Y^{\bullet}$ are isomorphic in $\mathscr{C}(A)$. In fact, if $f^{\bullet}: X^{\bullet} \rightarrow Y^{\bullet}$ and $g^{\bullet}: Y^{\bullet} \rightarrow X^{\bullet}$ are morphisms in $\mathscr{C}(A)$ such that $f^{\bullet} g^{\bullet}-1$ is null-homotopic, then there is a map $s^{i}: X^{i} \rightarrow X^{i-1}$ such that $f^{i} g^{i}-1=d_{X}^{i} s^{i+1}+s^{i} d_{X}^{i-1}$ for each $i$. The conclusion now follows from the fact that $d_{X}^{i}$ and $d_{Y}^{i}$ are radical maps for all $i$.

(c) For a radical complex $X^{\bullet}=\left(X^{i}, d^{i}\right) \in \mathscr{C}^{b}(A)$ with all terms projective, if $\operatorname{Hom}_{\mathscr{D}^{\mathrm{b}}(A)}\left(X^{\bullet}, A[m]\right)=0$ for all $m>0$, then $X^{i}=0$ for all $i<0$. Indeed, we may suppose that there is a maximal integer $t \geq 0$ such that $X^{-t} \neq 0$. Then there is a homomorphism $f: X^{-t} \rightarrow A$, which is not a radical map. Since $X^{\bullet}$ is a radical complex, we see that $f$, considered as a chain map from $X^{\bullet}$ to $A[t]$, is nonzero in $\mathscr{K}^{\mathrm{b}}(A)$; this contradicts our assumption. Thus, (c) holds. Similarly, we can prove the following.

(d) For a radical complex $Y^{\bullet}=\left(Y^{i}, d^{i}\right) \in \mathscr{C}^{b}(A)$ with all terms projective, if $\operatorname{Hom}_{\mathscr{D}^{\mathrm{b}}(A)}\left(A, Y^{\bullet}[m]\right)=0$ for all $m>n$, then $Y^{i}=0$ for all $i>n$.

Two algebras $A$ and $B$ are said to be derived-equivalent if their derived categories $\mathscr{D}^{\mathrm{b}}(A)$ and $\mathscr{D}^{\mathrm{b}}(B)$ are equivalent as triangulated categories. Rickard [14] proved that two algebras are derived-equivalent if and only if there is a complex $T^{\bullet}$ in $\mathscr{K}^{\mathrm{b}}(A$-proj) satisfying

(1) $\operatorname{Hom}_{\mathscr{D}^{\mathrm{b}}(A)}\left(T^{\bullet}, T^{\bullet}[n]\right)=0$ for all $n \neq 0$, and

(2) $\operatorname{add}\left(T^{\bullet}\right)$ generates $\mathscr{K}^{\mathrm{b}}(A$-proj) as a triangulated category,

such that $B \simeq \operatorname{End}_{\mathscr{D}^{\mathrm{b}}(A)}\left(T^{\bullet}\right)$. A complex in $\mathscr{K}^{\mathrm{b}}(A$-proj$)$ satisfying the above two conditions is called a tilting complex over $A$. By condition (2), each indecomposable projective $A$-module is a direct summand of $T^{i}$ for some integer $i$. It is known that, given a derived equivalence $F$ between $A$ and $B$, there is a unique (up to isomorphism) tilting complex $T^{\bullet}$ over $A$ such that $F\left(T^{\bullet}\right) \simeq B$. This complex $T^{\bullet}$ is called a tilting complex associated to $F$.

Let $F$ be a derived equivalence between two Artin algebras $A$ and $B$, and let $Q^{\bullet}$ be a tilting complex associated to $F$. Without loss of generality, we may assume that $Q^{\bullet}$ is radical, that $Q^{i}=0$ for $i<-n$ and $i>0$, and that 
$Q^{0} \neq 0 \neq Q^{-n}$. Then we have the following fact; for the convenience of the reader, we include here a proof.

Lemma 2.1. Let $A$ and $B$ be two Artin algebras, and let $F$ and $Q^{\bullet}$ be as above. If $G: \mathscr{D}^{\mathrm{b}}(B) \longrightarrow \mathscr{D}^{\mathrm{b}}(A)$ is a quasi-inverse of $F$, then there is a radical tilting complex $\bar{Q}^{\bullet}$ associated to $G$ of the following form:

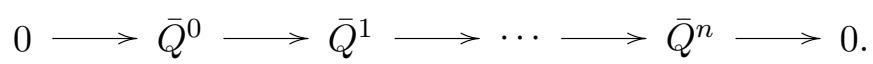

Proof. Note that the tilting complex $Q^{\bullet}$ associated to $F$ is radical and of the form

$$
0 \longrightarrow Q^{-n} \longrightarrow \cdots \longrightarrow Q^{-1} \longrightarrow Q^{0} \longrightarrow 0
$$

Let $\bar{Q}^{\bullet}$ be a radical complex in $\mathscr{K}^{\mathrm{b}}\left(B\right.$-proj) such that $\bar{Q}^{\bullet}$ is isomorphic to $F(A)$ in $\mathscr{D}^{\mathrm{b}}(B)$. Then $G\left(\bar{Q}^{\bullet}\right) \simeq G F(A) \simeq A$ in $\mathscr{D}^{\mathrm{b}}(A)$, which means that $\bar{Q}^{\bullet}$ is a tilting complex associated to $G$. Moreover, on the one hand, we have

$$
\operatorname{Hom}_{\mathscr{D}^{\mathrm{b}}(B)}\left(\bar{Q}^{\bullet}, B[m]\right) \simeq \operatorname{Hom}_{\mathscr{D}^{\mathrm{b}}(A)}\left(A, Q^{\bullet}[m]\right)=0
$$

for all $m>0$, and consequently, $\bar{Q}^{\bullet}$ has zero terms in all negative degrees. On the other hand, we have

$$
\operatorname{Hom}_{\mathscr{D} \mathrm{b}(B)}\left(B, \bar{Q}^{\bullet}[m]\right) \simeq \operatorname{Hom}_{\mathscr{D} \mathrm{b}(A)}\left(Q^{\bullet}, A[m]\right)=0
$$

for all $m>n$ and $\operatorname{Hom}_{\mathscr{D}^{\mathrm{b}}(B)}\left(B, \bar{Q}^{\bullet}[n]\right) \neq 0$. Thus, the complex $\bar{Q}^{\bullet}$ has zero terms in all degrees larger than $n$, and its $n$th term is nonzero.

The following lemma will be used frequently in our proofs. Again, we include here a proof for the convenience of the reader.

LEMMA 2.2. Let $A$ be an arbitrary ring, and let $A$-Mod be the category of all left (not necessarily finitely generated) A-modules. Suppose that $X^{\bullet}$ is a complex over A-Mod bounded above, and suppose that $Y^{\bullet}$ is a complex over A-Mod bounded below. Let $m$ be an integer. If one of the following two conditions holds:

(1) $X^{i}$ is projective for all $i>m$ and $Y^{j}=0$ for all $j<m$;

(2) $Y^{j}$ is injective for all $j<m$ and $X^{i}=0$ for all $i>m$,

then $\theta_{X \bullet, Y} \bullet: \operatorname{Hom}_{\mathscr{K}(A \text {-Mod })}\left(X^{\bullet}, Y^{\bullet}\right) \rightarrow \operatorname{Hom}_{\mathscr{D}(A \text {-Mod })}\left(X^{\bullet}, Y^{\bullet}\right)$ induced by the localization functor $\theta: \mathscr{K}(A$-Mod $) \rightarrow \mathscr{D}(A$-Mod $)$ is an isomorphism. 
Proof. For simplicity, we write $\mathscr{K}=\mathscr{K}(A$-Mod $)$ and $\mathscr{D}=\mathscr{D}(A$-Mod $)$. The category of all left (not necessarily finitely generated) projective $A$ modules is denoted by $A$-Proj. By applying the shift functor, we may assume that $m=0$. Suppose that (1) is satisfied.

First, we consider the case $X^{i}=0$ for all $i<0$. Let

$$
\cdots \longrightarrow P^{-1} \longrightarrow P^{0} \stackrel{\pi}{\longrightarrow} X^{0} \longrightarrow 0
$$

be a projective resolution of $X^{0}$. Then the complex

$$
\cdots \longrightarrow P^{-1} \longrightarrow P^{0} \stackrel{\pi d_{X}^{0}}{\longrightarrow} X^{1} \longrightarrow X^{2} \longrightarrow \cdots,
$$

denoted by $P_{X}^{\bullet}$, is in $\mathscr{K}\left(A\right.$-Proj) and bounded above since $X^{i}$ is projective for all $i>0$ by our assumption, and there is a quasi-isomorphism $\pi^{\bullet}: P_{X}^{\bullet} \rightarrow X^{\bullet}$ :

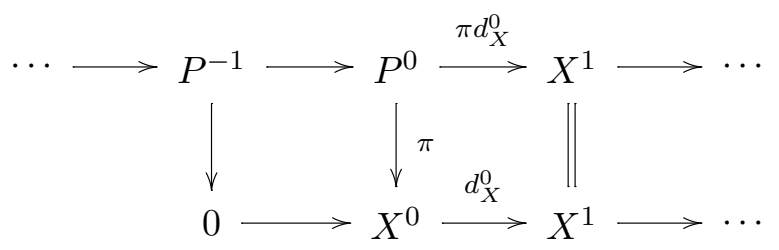

We claim that $\operatorname{Hom}_{\mathscr{K}}\left(\pi^{\bullet}, Y^{\bullet}\right): \operatorname{Hom}_{\mathscr{K}}\left(X^{\bullet}, Y^{\bullet}\right) \rightarrow \operatorname{Hom}_{\mathscr{K}}\left(P_{X}^{\bullet}, Y^{\bullet}\right)$ induced by $\pi^{\bullet}$ is an isomorphism. Actually, if $f^{\bullet} \in \operatorname{Hom}_{\mathscr{K}}\left(P_{X}^{\bullet}, Y^{\bullet}\right)$, then $f^{0}$ factorizes through the map $\pi: P^{0} \rightarrow X^{0}$. Suppose that $f^{0}=\pi g^{0}$ for some $g^{0}: X^{0} \rightarrow Y^{0}$. Let $g^{i}:=f^{i}$ for all $i>0$. Then $g^{\bullet}=\left(g^{i}\right)$ is a chain map from $X^{\bullet}$ to $Y^{\bullet}$ and $f^{\bullet}=\pi^{\bullet} g^{\bullet}$. Consequently, the map $\operatorname{Hom}_{\mathscr{K}}\left(\pi^{\bullet}, Y^{\bullet}\right)$ is surjective. Further, we shall show that the map $\operatorname{Hom}_{\mathscr{K}}\left(\pi^{\bullet}, Y^{\bullet}\right)$ is injective. In fact, if $\pi^{\bullet} \alpha^{\bullet}=0$ for a morphism $\alpha^{\bullet}$ in $\operatorname{Hom}_{\mathscr{K}}\left(X^{\bullet}, Y^{\bullet}\right)$, then there is map $h^{i}: X^{i} \rightarrow Y^{i-1}$ for all integers $i \geq 1$ such that $\pi \alpha^{0}=\pi d_{X}^{0} h^{1}$ and $\alpha^{i}=d_{X}^{i} h^{i+1}+h^{i} d_{Y}^{i-1}$ for all $i>0$. Thus, $\alpha^{0}=d_{X}^{0} h^{1}$ since $\pi$ is an epimorphism. Hence, $\alpha^{\bullet}=0$, which implies that $\operatorname{Hom}_{\mathscr{K}}\left(\pi^{\bullet}, Y^{\bullet}\right)$ is injective. It follows that $\operatorname{Hom}_{\mathscr{K}}\left(\pi^{\bullet}, Y^{\bullet}\right)$ is an isomorphism.

Note that $\pi^{\bullet}$ induces a commutative diagram:

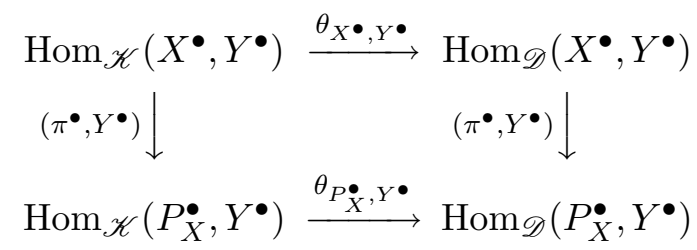


Since $\pi^{\bullet}$ is a quasi-isomorphism, the right vertical map is an isomorphism. We have shown that the left vertical map is an isomorphism. Moreover, since $P_{X}^{\bullet}$ is a complex in $\mathscr{K}\left(A\right.$-Proj) and bounded above, the map $\theta_{P_{X}^{\bullet}, Y^{\bullet}}$ is an isomorphism. It follows that $\theta_{X \bullet, Y} \bullet: \operatorname{Hom}_{\mathscr{K}}\left(X^{\bullet}, Y^{\bullet}\right) \longrightarrow \operatorname{Hom}_{\mathscr{D}}\left(X^{\bullet}, Y^{\bullet}\right)$ is an isomorphism.

Now, let $X^{\bullet}$ be an arbitrary complex satisfying condition (1). Then there is a distinguished triangle

$$
\sigma_{<0} X^{\bullet}[-1] \longrightarrow \sigma_{\geq 0} X^{\bullet} \stackrel{p}{\longrightarrow} X^{\bullet} \longrightarrow \sigma_{<0} X^{\bullet}
$$

in $\mathscr{K}$. This triangle can be viewed as a distinguished triangle in $\mathscr{D}$. Applying the functors $\operatorname{Hom}_{\mathscr{K}}\left(-, Y^{\bullet}\right)$ and $\operatorname{Hom}_{\mathscr{D}}\left(-, Y^{\bullet}\right)$ to this triangle, we have an exact commutative diagram

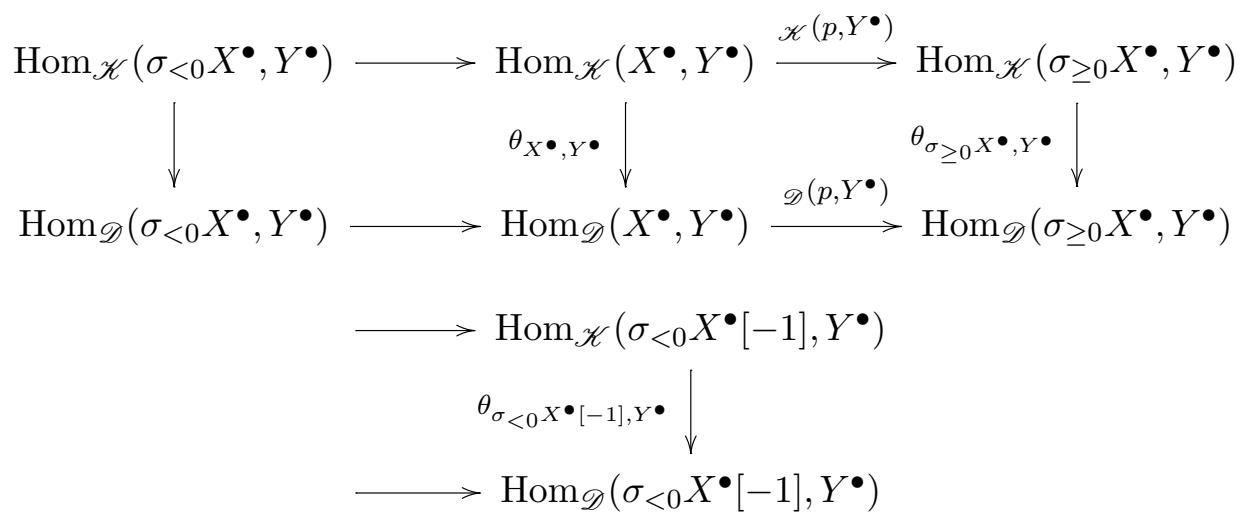

By our assumption, we have $Y^{i}=0$ for all $i<0$, and therefore $\operatorname{Hom}_{\mathscr{K}}\left(\sigma_{<0} X^{\bullet}\right.$, $\left.Y^{\bullet}\right)=0$. Note that $\sigma_{<0} X^{\bullet}$ is isomorphic in $\mathscr{D}$ to a complex $P_{1}^{\bullet}$ in $\mathscr{K}(A$-Proj $)$ such that $P_{1}^{i}=0$ for all $i \geq 0$. It follows that $\operatorname{Hom}_{\mathscr{D}}\left(\sigma_{<0} X^{\bullet}, Y^{\bullet}\right) \simeq \operatorname{Hom}_{\mathscr{D}}\left(P_{1}^{\bullet}\right.$, $\left.Y^{\bullet}\right) \simeq \operatorname{Hom}_{\mathscr{K}}\left(P_{1}^{\bullet}, Y^{\bullet}\right)=0$. Thus, both maps $\mathscr{K}\left(p, Y^{\bullet}\right)$ and $\mathscr{D}\left(p, Y^{\bullet}\right)$ are injective. Note that $\sigma_{\geq 0} X^{\bullet}$ is a complex satisfying condition (1) and that the terms of $\sigma_{\geq 0} X^{\bullet}$ in all negative degrees are zero. By the first part of the proof, we see that the map $\theta_{\sigma_{\geq 0} X} \bullet Y^{\bullet}$ is an isomorphism. It follows that $\theta_{X} \bullet Y^{\bullet}$ is injective. In particular, the map $\theta_{\sigma_{<0} X} \bullet[-1], Y \bullet$ is injective. By the five lemma (see [18, Exercise 1.3.3, page 13]), the map $\theta_{X} \bullet Y^{\bullet}$ is surjective. Thus, $\theta_{X \bullet, Y} \bullet$ is an isomorphism.

The proof for situation (2) proceeds similarly.

Remark. Suppose that $X^{\bullet}$ and $Y^{\bullet}$ are given as in Lemma 2.2. It follows from Lemma 2.2 that if the pair $\left(X^{\bullet}, Y^{\bullet}\right)$ satisfies one of the conditions 
in Lemma 2.2 and if the pair $\left(Y^{\bullet}, X^{\bullet}\right)$ satisfies one of the conditions in Lemma 2.2, then an isomorphism of $X^{\bullet}$ with $Y^{\bullet}$ in $\mathscr{D}(A$-Mod) induces an isomorphism of $X^{\bullet}$ with $Y^{\bullet}$ in $\mathscr{K}(A$-Mod $)$.

In the following, we point out a relationship between the Nakayama functor and a derived equivalence. Let $F: \mathscr{D}^{\mathrm{b}}(A) \longrightarrow \mathscr{D}^{\mathrm{b}}(B)$ be a derived equivalence between Artin algebras $A$ and $B$ over a commutative Artin ring $R$. By [14, Theorem 6.4], $F$ induces an equivalence from $\mathscr{K}^{\mathrm{b}}(A$-proj) to $\mathscr{K}^{\mathrm{b}}\left(B\right.$-proj). Note that the Nakayama functor $\nu_{A}: A$-proj $\longrightarrow A$-inj induces an equivalence from $\mathscr{K}^{\mathrm{b}}(A$-proj $)$ to $\mathscr{K}^{\mathrm{b}}(A$-inj $)$, which is again denoted by $\nu_{A}$. When $A$ and $B$ are finite-dimensional algebras over a field $k$, Rickard [15] shows that $F\left(\nu_{A} P^{\bullet}\right) \simeq \nu_{B} F\left(P^{\bullet}\right)$ in $\mathscr{D}^{\mathrm{b}}(B)$ for $P^{\bullet} \in \mathscr{K}^{\mathrm{b}}(A$-proj $)$. We can prove this isomorphism for Artin algebras by using the notion of AuslanderReiten triangle. Recall that a distinguished triangle $X \stackrel{f}{\longrightarrow} M \stackrel{g}{\longrightarrow} Y \stackrel{w}{\longrightarrow}$ $X[1]$ in $\mathscr{D}^{\mathrm{b}}(A)$ is called an Auslander-Reiten triangle if

(AR1) $X$ and $Y$ are indecomposable,

(AR2) $w \neq 0$, and

(AR3) if $t: U \longrightarrow Y$ is not a split epimorphism, then $t w=0$.

For a given object $Y$ in $\mathscr{D}^{\mathrm{b}}(A)$, if there is an Auslander-Reiten triangle $X \stackrel{f}{\longrightarrow} M \stackrel{g}{\longrightarrow} Y \stackrel{w}{\longrightarrow} X[1]$, then it is unique up to isomorphism [5, Proposition 4.3, page 33].

The first statement of the next lemma is essentially due to Happel (see [5, Theorem 4.6, page 37]), where the case of finite-dimensional algebras was considered. The second statement follows from the uniqueness of an Auslander-Reiten triangle ending at $Y$.

Lemma 2.3. Let $A$ be an Artin algebra over a commutative Artin ring R. Then, for each indecomposable complex $P^{\bullet}$ in $\mathscr{K}^{\mathrm{b}}(A$-proj), there is an Auslander-Reiten triangle

$$
\left(\nu_{A} P^{\bullet}\right)[-1] \longrightarrow L^{\bullet} \longrightarrow P^{\bullet} \longrightarrow \nu_{A} P^{\bullet}
$$

in $\mathscr{D}^{\mathrm{b}}(A)$. Furthermore, we have $F\left(\nu_{A} P^{\bullet}\right) \simeq \nu_{B} F\left(P^{\bullet}\right)$ in $\mathscr{D}^{\mathrm{b}}(B)$.

Finally, let us remark that, given a functor $F: \mathcal{C} \rightarrow \mathcal{D}$, if we fix an object $F_{X}$ in $\mathcal{D}$ for each object $X$ in $\mathcal{C}$ such that $F_{X} \simeq F(X)$, then there is a functor $F^{\prime}: \mathcal{C} \rightarrow \mathcal{D}$ such that $F^{\prime} \simeq F$ and $F^{\prime}(X)=F_{X}$ for every $X$ in $\mathcal{C}$. Actually, let $s_{X}$ denote a fixed isomorphism from $F_{X}$ to $F(X)$ for each $X$, and define $F^{\prime}(f):=s_{X} F(f) s_{Y}^{-1}$ for each $f: X \rightarrow Y$. Then $F^{\prime}$ is a desired functor. 


\section{$\S 3$. Stable equivalences induced by derived equivalences}

In this section, we first construct a functor $\bar{F}: A$ - $\underline{\bmod } \longrightarrow B$ - $\underline{\bmod }$ between the stable module categories of two Artin algebras $A$ and $B$ from a given derived equivalence $F: \mathscr{D}^{\mathrm{b}}(A) \longrightarrow \mathscr{D}^{\mathrm{b}}(B)$, and then we give a sufficient condition to ensure that the functor $\bar{F}$ is an equivalence. In Section 5 , we shall see a stronger conclusion when we work with finite-dimensional algebras instead of general Artin algebras.

Let us first recall some notions and notations. Let $A$ be an Artin algebra. The homotopy category $\mathscr{K}^{\mathrm{b}}(A$-proj) can be considered as a triangulated full subcategory of $\mathscr{D}^{\mathrm{b}}(A)$. Let $\mathscr{D}^{\mathrm{b}}(A) / \mathscr{K}^{\mathrm{b}}(A$-proj) be the Verdier quotient of $\mathscr{D}^{\mathrm{b}}(A)$ by the subcategory $\mathscr{K}^{\mathrm{b}}(A$-proj). (For the definition, we refer the reader to the excellent book [11].) Then there is a canonical functor $\Sigma^{\prime}: A$-mod $\longrightarrow \mathscr{D}^{\mathrm{b}}(A) / \mathscr{K}^{\mathrm{b}}(A$-proj$)$ obtained by composing the natural embedding of $A$-mod into $\mathscr{D}^{\mathrm{b}}(A)$ with the quotient functor from $\mathscr{D}^{\mathrm{b}}(A)$ to $\mathscr{D}^{\mathrm{b}}(A) / \mathscr{K}^{\mathrm{b}}(A$-proj $)$. Clearly, $\Sigma^{\prime}(P)$ is isomorphic to zero for each projective $A$-module $P$, so $\Sigma^{\prime}$ factorizes through the canonical functor from $A$-mod to $A$-mod. This gives rise to an additive functor $\Sigma: A$-mod $\longrightarrow$ $\mathscr{D}^{\mathrm{b}}(A) / \mathscr{K}^{\mathrm{b}}(A$-proj $)$.

Rickard [13] proved that $\Sigma$ is an equivalence provided that the algebra $A$ is self-injective. But for an arbitrary algebra, this is no longer true in general; for instance, if $A$ is a nonsemisimple Artin algebra of finite global dimension, then the quotient category $\mathscr{D}^{\mathrm{b}}(A) / \mathscr{K}^{\mathrm{b}}(A$-proj $)$ is zero, and therefore the functor $\Sigma$ is a zero functor, which cannot be an equivalence.

Let $A$ and $B$ be two Artin algebras. Suppose that $F: \mathscr{D}^{\mathrm{b}}(A) \longrightarrow \mathscr{D}^{\mathrm{b}}(B)$ is a derived equivalence between $A$ and $B$. Then $F$ induces an equivalence between the quotient categories $\mathscr{D}^{\mathrm{b}}(A) / \mathscr{K}^{\mathrm{b}}(A$-proj$)$ and $\mathscr{D}^{\mathrm{b}}(B) /$ $\mathscr{K}^{\mathrm{b}}(B$-proj). For simplicity, we denote this induced equivalence also by $F$. Thus, if $A$ and $B$ are self-injective, then $A$-mod and $B$-mod are equivalent. However, this is not true in general for arbitrary finite-dimensional algebras; namely, we cannot get an equivalence of stable module categories from a given derived equivalence in general. Nevertheless, we may ask if there is any "good" functor $\bar{F}: A$-mod $\longrightarrow B$ - $\underline{\bmod }$ induced by $F$, which could be a possible candidate for a stable equivalence under certain additional conditions and would cover the most interesting known situations.

In the following, we shall construct an additive functor $\bar{F}: A$ - $\underline{\bmod } \longrightarrow$ $B$-mod from $F$ such that the diagram 


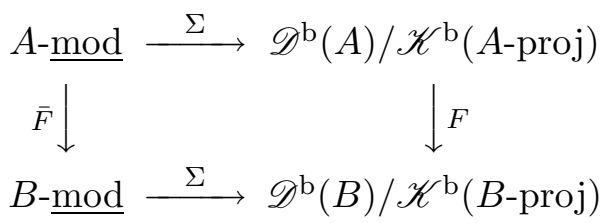

of additive functors is commutative up to natural isomorphism. Furthermore, we shall construct a possible candidate for the inverse of $\bar{F}$ under an additional condition.

From now on, $A$ and $B$ are Artin $R$-algebras and $F$ is a derived equivalence between $A$ and $B$ with a quasi-inverse $G$. Let $Q^{\bullet}$ be a tilting complex over $A$ associated to $F$ of the form

$$
Q^{\bullet}: 0 \longrightarrow Q^{-n} \longrightarrow \cdots \longrightarrow Q^{-1} \longrightarrow Q^{0} \longrightarrow 0
$$

such that all differentials are radical maps. By Lemma 2.1, there is a tilting complex $\bar{Q}^{\bullet}$ associated to $G$ of the form

$$
\bar{Q}^{\bullet}: 0 \longrightarrow \bar{Q}^{0} \longrightarrow \bar{Q}^{1} \longrightarrow \cdots \longrightarrow \bar{Q}^{n} \longrightarrow 0
$$

with all differentials being radical maps. We define $Q=\bigoplus_{i=1}^{n} Q^{-i}$ and $\bar{Q}=$ $\bigoplus_{i=1}^{n} \bar{Q}^{i}$. Furthermore, we assume throughout the article that $Q^{0} \neq 0 \neq$ $Q^{-n}$.

Lemma 3.1. Let $X$ be an A-module. Then $F(X)$ is isomorphic in $\mathscr{D}^{\mathrm{b}}(B)$ to a radical complex $\bar{Q}_{X}^{\bullet}$ of the form

$$
0 \longrightarrow \bar{Q}_{X}^{0} \longrightarrow \bar{Q}_{X}^{1} \longrightarrow \cdots \longrightarrow \bar{Q}_{X}^{n} \longrightarrow 0
$$

with $\bar{Q}_{X}^{i} \in \operatorname{add}\left({ }_{B} \bar{Q}\right)$ for all $i=1,2, \ldots, n$. Moreover, the complex $\bar{Q}_{X}^{\bullet}$ of this form is unique up to isomorphism in $\mathscr{C}^{b}(B)$. In particular, if $X$ is projective, then $\bar{Q}_{X}^{\bullet}$ is isomorphic in $\mathscr{C}^{b}(B)$ to a complex in $\operatorname{add}\left(\bar{Q}^{\bullet}\right)$.

Proof. Let $H^{i}$ be the $i$ th cohomology functor on complexes. First of all, we have $H^{i}(F(X)) \simeq \operatorname{Hom}_{\mathscr{D}^{\mathrm{b}}(B)}(B, F(X)[i]) \simeq \operatorname{Hom}_{\mathscr{D}^{\mathrm{b}}(A)}\left(Q^{\bullet}, X[i]\right)=0$ for all $i>n$ and all $i<0$, which means that $F(X)$ has trivial homology in negative degrees and degrees larger than $n$. Clearly, we may assume that $X$ is indecomposable.

If $X$ is projective, then $X$ is isomorphic to a direct summand of $A$. Consequently, $F(X)$ is isomorphic in $\mathscr{D}^{\mathrm{b}}(B)$ to a direct summand $L^{\bullet}$ of the 
complex $\bar{Q}^{\bullet}$. Since all terms of $\bar{Q}^{\bullet}$ in positive degrees are in $\operatorname{add}\left({ }_{B} \bar{Q}\right)$, all terms of $L^{\bullet}$ in positive degrees are in $\operatorname{add}\left({ }_{B} \bar{Q}\right)$. This shows that for every projective $A$-module $P$, the complex $F(P)$ is isomorphic in $\mathscr{D}^{\mathrm{b}}(B)$ to a complex with all of its terms in positive degrees in $\operatorname{add}\left({ }_{B} \bar{Q}\right)$. Now we show that if $P^{\bullet}$ is a complex in $\mathscr{K}^{\mathrm{b}}\left(A\right.$-proj) with $P^{i}=0$ for all $i>0$, then $F\left(P^{\bullet}\right)$ is isomorphic in $\mathscr{D}^{\mathrm{b}}(B)$ to a complex in which all of its terms in positive degrees belong to $\operatorname{add}\left({ }_{B} \bar{Q}\right)$. In fact, if $P^{\bullet}$ has only one nonzero term, then we may write $P^{\bullet}=P[t]$ for a projective $A$-module $P$ and a nonnegative integer $t$. In this case, $F\left(P^{\bullet}\right)$ is isomorphic to a direct summand of $\bar{Q}^{\bullet}[t]$ in which all terms in positive degrees are in $\operatorname{add}\left({ }_{B} \bar{Q}\right)$, as desired. Now, we assume that $P^{\bullet}$ has at least two nonzero terms. Then there is an integer $s<0$ such that the brutal truncations $\sigma_{<s} P^{\bullet}$ and $\sigma_{\geq s} P^{\bullet}$ have fewer nonzero terms than $P^{\bullet}$ does. By induction, the complexes $F\left(\sigma_{<s} P^{\bullet}\right)$ and $F\left(\sigma_{\geq s} P^{\bullet}\right)$ are respectively isomorphic to complexes $Y^{\bullet}$ and $Z^{\bullet}$ in $\mathscr{K}^{\mathrm{b}}(A$-proj $)$, such that their terms in all positive degrees are in $\operatorname{add}\left({ }_{B} \bar{Q}\right)$. Since $P^{\bullet}$ is the mapping cone of the map $d_{P}^{s-1}$ from $\left(\sigma_{<s} P^{\bullet}\right)[-1]$ to $\sigma_{\geq s} P^{\bullet}$, the complex $F\left(P^{\bullet}\right)$ is isomorphic to the mapping cone of a chain map from $Y^{\bullet}[-1]$ to $Z^{\bullet}$, and consequently, all of its terms in positive degrees lie in $\operatorname{add}\left({ }_{B} \bar{Q}\right)$.

Now, suppose that $X$ is an arbitrary indecomposable $A$-module and suppose that $P^{\bullet}=\left(P^{i}, d^{i}\right)$ is a minimal projective resolution of $X$. We denote by $\Omega^{n}(X)$ the $n$th syzygy of $X$ and by $P_{1}^{\bullet}$ the complex

$$
0 \longrightarrow P^{-n+1} \longrightarrow P^{-n+2} \longrightarrow \cdots \longrightarrow P^{0} \longrightarrow 0 \text {. }
$$

Then we have a distinguished triangle in $\mathscr{D}^{\mathrm{b}}(A)$ :

$$
\Omega^{n}(X)[n-1] \longrightarrow P_{1}^{\bullet} \longrightarrow X \longrightarrow \Omega^{n}(X)[n]
$$

From this triangle one gets the following distinguished triangle in $\mathscr{D}^{\mathrm{b}}(B)$ :

$$
F\left(\Omega^{n}(X)\right)[n-1] \longrightarrow F\left(P_{1}^{\bullet} \longrightarrow F(X) \longrightarrow F\left(\Omega^{n}(X)\right)[n]\right.
$$

The complex $P_{1}^{\bullet}$ is in $\mathscr{K}^{\mathrm{b}}(A$-proj $)$, and all the terms of $P_{1}^{\bullet}$ in positive degrees are zero. Hence, $F\left(P_{1}^{\bullet}\right)$ is isomorphic to a complex $Q_{1}^{\bullet}$ in $\mathscr{K}^{\mathrm{b}}(B$-proj $)$ with $Q_{1}^{i}$ in $\operatorname{add}\left({ }_{B} \bar{Q}\right)$ for all $i>0$. Since $\Omega^{n}(X)$ is an $A$-module, the complex $F\left(\Omega^{n}(X)\right)$ has trivial homology in all degrees larger than $n$. Thus, the complex $F\left(\Omega^{n}(X)\right)$ is isomorphic in $\mathscr{D}(B)$ to a complex $P_{2}^{\bullet} \in \mathscr{K}^{-}(B$-proj) with zero terms in all degrees larger than $n$. It follows that $P_{2}^{\bullet}[n-1]$ has 
zero terms in all degrees larger than 1 . Hence, $F(X)$ is isomorphic to the mapping cone $\operatorname{con}(\mu)$ of a map $\mu$ from $P_{2}^{\bullet}[n-1]$ to $Q_{1}^{\bullet}$, and all the terms of $\operatorname{con}(\mu)$ in positive degrees are in $\operatorname{add}\left({ }_{B} \bar{Q}\right)$. Note that $F(X)$ has zero homology in all negative degrees and degrees larger than $n$. Thus, $\operatorname{con}(\mu)$ has the same homology property and is isomorphic in $\mathscr{D}(B)$ to a radical complex

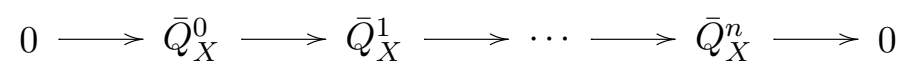

with $\bar{Q}_{X}^{i} \in \operatorname{add}\left({ }_{B} \bar{Q}\right)$ for all $i=1,2, \ldots, n$.

Suppose that $U^{\bullet}$ and $V^{\bullet}$ are two radical complexes of the form in Lemma 3.1 such that both $U^{\bullet}$ and $V^{\bullet}$ are isomorphic to $F(X)$ in $\mathscr{D}^{\mathrm{b}}(B)$. Then $U^{\bullet}$ and $V^{\bullet}$ are isomorphic in $\mathscr{K}^{\mathrm{b}}(B)$ by Lemma 2.2 . Since $U^{\bullet}$ and $V^{\bullet}$ are radical complexes, we know that $U^{\bullet}$ and $V^{\bullet}$ are isomorphic as complexes.

If $X$ is projective, then $X \in \operatorname{add}(A)$ and $F(X) \in \operatorname{add}(F(A))$. Since $F(A) \simeq$ $F G\left(\bar{Q}^{\bullet}\right) \simeq \bar{Q}^{\bullet}$ in $\mathscr{D}^{\mathrm{b}}(B)$, we see that $F(X)$ is isomorphic in $\mathscr{K}^{\mathrm{b}}(B$-proj) to a complex $Y^{\bullet} \in \operatorname{add}\left(\bar{Q}^{\bullet}\right)$. Thus, $\bar{Q}_{X}^{\bullet}$ is isomorphic in $\mathscr{D}^{\mathrm{b}}(B)$ to $Y^{\bullet}$. Since $Y^{\bullet}$ is a complex with the properties in Lemma 3.1, we have $Y^{\bullet} \simeq \bar{Q}_{X}^{\bullet}$ as complexes by the uniqueness of $\bar{Q}_{X}^{\bullet}$. This shows that $\bar{Q}_{X}^{\bullet} \in \operatorname{add}\left(\bar{Q}^{\bullet}\right)$. Thus, Lemma 3.1 is proved.

Similarly, we have the following lemma.

Lemma 3.2. Let $U$ be a $B$-module. Then $G(U)$ is isomorphic in $\mathscr{D}^{\mathrm{b}}(A)$ to a radical complex $Q_{U}^{\bullet}$ of the form

$$
0 \longrightarrow Q_{U}^{-n} \longrightarrow \cdots \longrightarrow Q_{U}^{-1} \longrightarrow Q_{U}^{0} \longrightarrow 0
$$

with $Q_{U}^{-i} \in \operatorname{add}\left(\nu_{A} Q\right)$ for all $i=1,2, \ldots, n$. Moreover, the complex $Q_{U}^{\bullet}$ of this form is unique up to isomorphism in $\mathscr{C}^{b}(A)$.

Remark. One can easily see that if $X \simeq Y \oplus Z$ in $A$-mod, then the complex $\bar{Q}_{X}^{\bullet}$ defined in Lemma 3.1 is isomorphic in $\mathscr{C}^{b}(B)$ to the direct sum of $\bar{Q}_{Y}^{\bullet}$ and $\bar{Q}_{Z}^{\bullet}$. Similarly, if $U \simeq V \oplus W$ in $B$-mod, then the complex $Q_{U}^{\bullet}$ defined in Lemma 3.2 is isomorphic in $\mathscr{C}^{b}(A)$ to the direct sum of $Q_{V}^{\bullet}$ and $Q_{W}^{\bullet}$.

The next lemma is useful in our proofs.

Lemma 3.3. Let $A$ be an Artin algebra, and let $f: X \longrightarrow Y$ be a homomorphism between two A-modules $X$ and $Y$. Suppose that $P^{\bullet}$ is a complex 
in $\mathscr{K}^{\mathrm{b}}(A)$ with $P^{i}$ projective for all $i \geq 0$ and injective for all $i<0$. If $f$ factorizes in $\mathscr{D}^{\mathrm{b}}(A)$ through $P^{\bullet}$, then $f$ factorizes through a projective A-module.

Proof. Suppose that

$$
f=g h \quad \text { for } g \in \operatorname{Hom}_{\mathscr{D}^{\mathrm{b}}(A)}\left(X, P^{\bullet}\right) \text { and } h \in \operatorname{Hom}_{\mathscr{D}^{\mathrm{b}}(A)}\left(P^{\bullet}, Y\right) .
$$

By Lemma 2.2(2), we have $g=g^{\bullet}$ in $\mathscr{D}^{\mathrm{b}}(A)$ for some chain map $g^{\bullet}$ from $X$ to $P^{\bullet}$, and by Lemma $2.2(1)$, we get $h=h^{\bullet}$ in $\mathscr{D}^{\mathrm{b}}(A)$ for some chain map $h^{\bullet}$ from $P^{\bullet}$ to $Y$. Hence, $f=g^{0} h^{0}$ in $\mathscr{D}^{\mathrm{b}}(A)$. Since $A$-mod is fully embedded in $\mathscr{D}^{\mathrm{b}}(A)$, we see that $f=g^{0} h^{0}$ factorizes through the projective $A$-module $P^{0}$ in $A$-mod.

Now we define the functor $\bar{F}$. Pick an $A$-module $X$; by Lemma 3.1, we know that $F(X)$ is isomorphic in $\mathscr{D}^{\mathrm{b}}(B)$ to a radical complex $\bar{Q}_{X}^{\bullet}$ of the form

$$
0 \longrightarrow \bar{Q}_{X}^{0} \longrightarrow \bar{Q}_{X}^{1} \longrightarrow \cdots \longrightarrow \bar{Q}_{X}^{n} \longrightarrow 0
$$

with $\bar{Q}_{X}^{i} \in \operatorname{add}\left({ }_{B} \bar{Q}\right)$ for all $i=1,2, \ldots, n$. From now on, for each $A$-module $X$, we choose (once and for all) such a complex $\bar{Q}_{X}^{\bullet}$. For each homomorphism $f: X \longrightarrow Y$, we denote by $f$ the image of $f$ under the canonical surjective map from $\operatorname{Hom}_{A}(X, Y)$ to $\underline{\operatorname{Hom}}_{A}(X, Y)$.

Proposition 3.4. Let $F: \mathscr{D}^{\mathrm{b}}(A) \longrightarrow \mathscr{D}^{\mathrm{b}}(B)$ be a derived equivalence between Artin algebras $A$ and $B$. Then there is an additive functor $\bar{F}$ : $A$-mod $\longrightarrow B$-mod sending $X$ to $\bar{Q}_{X}^{0}$ such that the diagram of additive functors

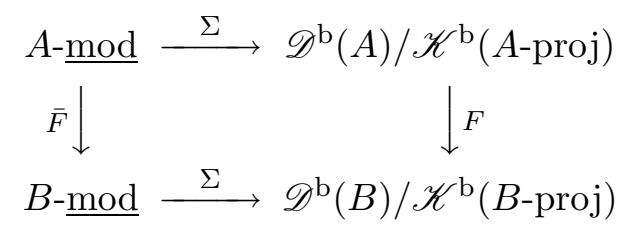

is commutative up to natural isomorphism.

Proof. By Lemma 3.1 and the remark at the end of Section 2, we may assume that $F(X)$ is just $\bar{Q}_{X}^{\bullet}$ for each $A$-module $X$, where $\bar{Q}_{X}^{\bullet}$ is the complex that we have fixed above. Let $\bar{Q}_{X}^{+}$denote the complex $\sigma_{\geq 1} \bar{Q}_{X}^{\bullet}$. Then we have a distinguished triangle in $\mathscr{D}^{\mathrm{b}}(B)$ :

$$
\bar{Q}_{X}^{+} \stackrel{i_{X}}{\longrightarrow} F(X) \stackrel{\pi_{X}}{\longrightarrow} \bar{Q}_{X}^{0} \stackrel{\alpha_{X}}{\longrightarrow} \bar{Q}_{X}^{+}[1]
$$


For each homomorphism $f: X \longrightarrow Y$ of $A$-modules $X$ and $Y$, there is a commutative diagram

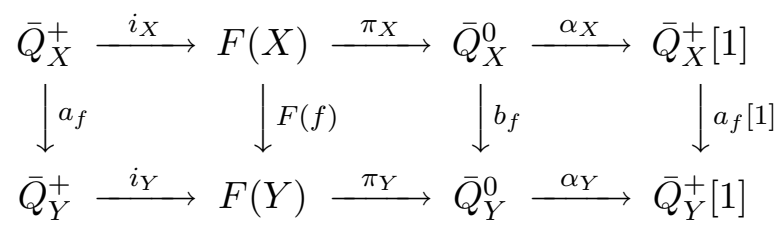

The map $a_{f}$ exists because $i_{X} F(f) \pi_{Y}$ belongs to $\operatorname{Hom}_{\mathscr{D}^{\mathrm{b}}(B)}\left(\bar{Q}_{X}^{+}, \bar{Q}_{Y}^{0}\right) \simeq$ $\operatorname{Hom}_{\mathscr{K}^{\mathrm{b}}(B)}\left(\bar{Q}_{X}^{+}, \bar{Q}_{Y}^{0}\right)=0$. Since $B$-mod is fully embedded in $\mathscr{D}(B)$, the morphism $b_{f}$ is a homomorphism of modules. If we have another $A$-module homomorphism $b_{f}^{\prime}$ such that $\pi_{X} b_{f}^{\prime}=F(f) \pi_{Y}$, then $\pi_{X}\left(b_{f}-b_{f}^{\prime}\right)=F(f) \pi_{Y}-$ $F(f) \pi_{Y}=0$ and $b_{f}-b_{f}^{\prime}$ factorizes through $\bar{Q}_{X}^{+}[1]$. By Lemma 3.3, the $B$-module homomorphism $b_{f}-b_{f}^{\prime}$ factorizes through a projective $B$-module. Thus, for each $A$-module homomorphism $f$ in $\operatorname{Hom}_{A}(X, Y)$, the morphism $b_{f}$ in $\underline{\operatorname{Hom}}_{B}\left(\bar{Q}_{X}^{0}, \bar{Q}_{Y}^{0}\right)$ is uniquely determined by $f$.

Suppose that $f: X \longrightarrow Y$ and $g: Y \longrightarrow Z$ are two homomorphisms of $A$-modules; we can see that $F(f g) \pi_{Z}=\pi_{X} b_{f g}$ and $F(f g) \pi_{Z}=\pi_{X}\left(b_{f} b_{g}\right)$. By the uniqueness of $\underline{b_{f g}}$, we have $\underline{b_{f g}}=\underline{b_{f}} \underline{b_{g}}$.

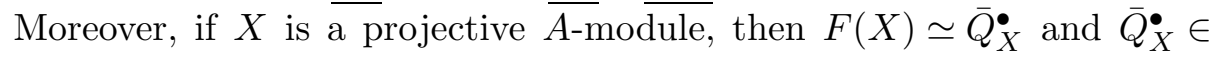
$\operatorname{add}\left(\bar{Q}^{\bullet}\right)$ by Lemma 3.1. In particular, $\bar{Q}_{X}^{0}$ is projective. Thus, if $f$ factorizes through a projective module $P$, say, $f=g h$ with $g \in \operatorname{Hom}_{A}(X, P)$ and $h \in$ $\operatorname{Hom}_{A}(P, Y)$, then $b_{f}$ factorizes through a projective $B$-module since $b_{f}=$ $b_{g h}=\left(b_{g h}-b_{g} b_{h}\right)+b_{g} b_{h}$ and since both $b_{g h}-b_{g} b_{h}$ and $b_{g} b_{h}$ factorize through projective $B$-modules.

For each $A$-module $X$, we define $\bar{F}(X)=\bar{Q}_{X}^{0}$. Note that $\bar{Q}_{X}^{0}$ is, up to isomorphism, uniquely determined by $X$ (see Lemma 3.1). For each homomorphism $\underline{f}$ in $\underline{\operatorname{Hom}}_{A}(X, Y)$, we set $\bar{F}(\underline{f})=b_{f}$. Then the above discussions show that $\overline{\bar{F}}$ is well defined on Hom-sets and that $\bar{F}$ is a functor from $A$-mod to $B$-mod. Note that $\bar{F}$ is additive since $F$ is additive.

To finish the proof of the proposition, it remains to show that $\pi_{X}: F(X) \longrightarrow$ $\bar{F}(X)$ is a natural isomorphism in the quotient category $\mathscr{D}^{\mathrm{b}}(B) / \mathscr{K}^{\mathrm{b}}(B$-proj $)$. That the morphism $\pi_{X}$ is an isomorphism follows from the fact that $\bar{Q}_{X}^{+}$is isomorphic to the zero object in $\mathscr{D}^{\mathrm{b}}(B) / \mathscr{K}^{\mathrm{b}}\left(B\right.$-proj). Clearly, $\pi_{X}$ is natural 
in $X$ since we have a commutative diagram

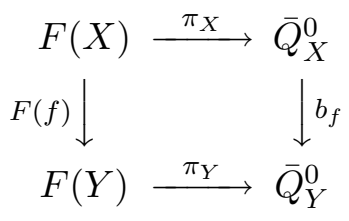

in the quotient category $\mathscr{D}^{\mathrm{b}}(B) / \mathscr{K}^{\mathrm{b}}(B$-proj $)$.

Now, it is appropriate to introduce a name for the functor $\bar{F}$. Given a derived equivalence $F$, the functor $\bar{F}$ constructed in Proposition 3.4 is called a stable functor of $F$ throughout this article.

Proposition 3.5. If $\operatorname{add}\left({ }_{A} Q\right)=\operatorname{add}\left(\nu_{A} Q\right)$, then there is an additive functor $\bar{G}: B$-mod $\longrightarrow A$-mod sending $U$ to $Q_{U}^{0}$ such that the diagram of additive functors

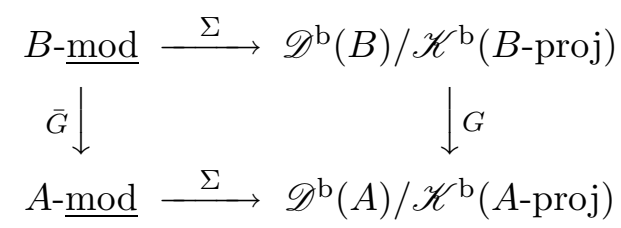

is commutative up to natural isomorphism.

Proof. The idea of the proof is similar to that of Proposition 3.4. We outline only the key points of the construction of $\bar{G}$.

For a $B$-module $U$, by Lemma $3.2, G(U)$ is isomorphic in $\mathscr{D}^{\mathrm{b}}(A)$ to a complex $Q_{U}^{\bullet}$ such that $Q_{U}^{i} \in \operatorname{add}\left(\nu_{A} Q\right)$ for all $i<0$ and $Q_{U}^{j}=0$ for all $j>0$. By the remark at the end of Section 2, we can assume that $G(U)$ is just $Q_{U}^{\bullet}$. Let $Q_{U}^{-}$denote the complex $\sigma_{<0} Q_{U}^{\bullet}$. We have a distinguished triangle in $\mathscr{D}^{\mathrm{b}}(A)$ :

$$
Q_{U}^{-}[-1] \stackrel{\beta_{U}}{\longrightarrow} Q_{U}^{0} \stackrel{\lambda_{U}}{\longrightarrow} G(U) \stackrel{\gamma_{U}}{\longrightarrow} Q_{U}^{-}
$$

Now, if $g: U \longrightarrow V$ is a homomorphism of $B$-modules, then we have a commutative diagram

$$
\begin{gathered}
Q_{U}^{-}[-1] \stackrel{\beta_{U}}{\longrightarrow} Q_{U}^{0} \stackrel{\lambda_{U}}{\longrightarrow} G(U) \stackrel{\gamma_{U}}{\longrightarrow} Q_{U}^{-} \\
\downarrow v_{g}[-1] \stackrel{u_{g}}{ } \\
Q_{V}^{-}[-1] \stackrel{\beta_{V}}{\longrightarrow} Q_{V}^{0} \stackrel{\lambda_{V}}{\longrightarrow} G(V) \stackrel{\gamma_{V}}{\longrightarrow} Q_{V}^{-}
\end{gathered}
$$


The existence of $u_{g}$ follows from the fact that the morphism $\lambda_{U} G(g) \gamma_{V}$ belongs to $\operatorname{Hom}_{\mathscr{D}^{\mathrm{b}}(A)}\left(Q_{U}^{0}, Q_{V}^{-}\right) \simeq \operatorname{Hom}_{\mathscr{K}^{\mathrm{b}}(A)}\left(Q_{U}^{0}, Q_{V}^{-}\right)=0$. Since $A$-mod is fully embedded in $\mathscr{D}(A)$, the map $u_{g}$ can be chosen to be an $A$-module homomorphism. Moreover, if $u_{g}^{\prime}: Q_{U}^{0} \longrightarrow Q_{V}^{0}$ is another morphism such that $u_{g}^{\prime} \lambda_{V}=\lambda_{U} G(g)$, then $\left(u_{g}-u_{g}^{\prime}\right) \lambda_{V}=0$ and $u_{g}-u_{g}^{\prime}$ factorizes through $Q_{V}^{-}[-1]$. Since $\operatorname{add}\left({ }_{A} Q\right)=\operatorname{add}\left(\nu_{A} Q\right)$, all the terms of the complex $Q_{V}^{-}[-1]$ are projective-injective. By Lemma 3.3, the morphism $u_{g}-u_{g}^{\prime}$ factorizes through a projective module. Thus, for each $B$-module homomorphism $g$, the morphism $\underline{u}_{g}$ in $\underline{\operatorname{Hom}}_{A}\left(Q_{U}^{0}, Q_{V}^{0}\right)$ is uniquely determined by $g$.

As in the proof of Proposition 3.4, we can show that the composition of two morphisms is preserved; namely, $\underline{u_{g h}}=\underline{u_{g}} \underline{u_{h}}$ for all $g \in \operatorname{Hom}_{B}(U, V)$ and all $h \in \operatorname{Hom}_{B}(V, W)$.

Moreover, if $P$ is a projective $B$-module, then $Q_{P}^{\bullet}$ is isomorphic in $\mathscr{D}^{\mathrm{b}}(A)$ to a complex $Q_{1}^{\bullet}$ in $\operatorname{add}\left(Q^{\bullet}\right)$. Since $\operatorname{add}\left({ }_{A} Q\right)=\operatorname{add}\left(\nu_{A} Q\right)$, the complex $Q_{1}^{\bullet}$ is of the form in Lemma 3.2. By the uniqueness of $Q_{P}^{\bullet}$, we have an isomorphism $Q_{P}^{\bullet} \simeq Q_{1}^{\bullet}$ in $\mathscr{C}^{b}(A)$. Hence, $Q_{P}^{0} \simeq Q_{1}^{0}$ and $Q_{P}^{0}$ is a projective $A$-module. Thus, if $g: U \longrightarrow V$ factorizes through a projective $B$-module $P$, that is, if $g=s t$ for $s: U \longrightarrow P$ and $t: P \longrightarrow V$, then $u_{g}=u_{s t}=\left(u_{s t}-u_{s} u_{t}\right)+u_{s} u_{t}$ factorizes through a projective $A$-module. This shows that the map $\underline{g} \mapsto \underline{u_{g}}$ is well defined.

Now, we define $\bar{G}(U):=Q_{U}^{0}$ for each $B$-module $U$ and $\bar{G}(\underline{g}):=\underline{u_{g}}$ for each morphism $g$ in $B$-mod. Note that $Q_{U}^{0}$ is, up to isomorphism, uniquely determined by $\bar{U}$ (see Lemma 3.2). Thus, we obtain an additive functor $\bar{G}$ from $B$-mod to $A$-mod. Moreover, the map $\lambda_{U}$ is a natural isomorphism in the quotient category $\mathscr{D}^{\mathrm{b}}(A) / \mathscr{K}^{\mathrm{b}}(A$-proj $)$ since $Q_{U}^{-}$is in $\mathscr{K}^{\mathrm{b}}(A$-proj) .

Proposition 3.6. Suppose that $\operatorname{add}\left({ }_{A} Q\right)=\operatorname{add}\left(\nu_{A} Q\right)$. Let $\bar{F}$ and $\bar{G}$ be the functors constructed in Proposition 3.4 and Proposition 3.5, respectively. Then the composition $\bar{G} \bar{F}$ is naturally isomorphic to the identity functor $1_{A-\text { mod. }}$. In particular, $\bar{G}$ is dense, and the restriction of $\bar{G}$ to $\operatorname{Im}(\bar{F})$ is full.

Proof. For each $A$-module $X$, we may assume that $F(X)$ is the complex $\bar{Q}_{X}^{\bullet}$ defined in Lemma 3.1. For each $B$-module $U$, we assume that $G(U)$ is the complex $Q_{U}^{\bullet}$ defined in Lemma 3.2. We set $\bar{Q}_{X}^{+}=\sigma_{\geq 1} \bar{Q}_{X}^{\bullet}$ and $Q_{U}^{-}=$ $\sigma_{<0} Q_{U}^{\bullet}$. Then all the terms of $Q_{U}^{-}$are projective-injective since $\operatorname{add}\left({ }_{A} Q\right)=$ $\operatorname{add}\left(\nu_{A} Q\right)$. By definition, we have $\bar{F}(X)=\bar{Q}_{X}^{0}$ for each $A$-module $X$ (see Proposition 3.4) and $\bar{G}(U)=Q_{U}^{0}$ for each $B$-module $U$ (see Proposition 3.5). 
Thus, for each $A$-module $X$, there is a distinguished triangle

$$
\bar{Q}_{X}^{+} \stackrel{i_{X}}{\longrightarrow} F(X) \stackrel{\pi_{X}}{\longrightarrow} \bar{F}(X) \stackrel{\alpha_{X}}{\longrightarrow} \bar{Q}_{X}^{+}[1]
$$

in $\mathscr{D}^{\mathrm{b}}(B)$ and a distinguished triangle

$$
Q_{\bar{F} X}^{-}[-1] \stackrel{\beta_{\bar{F} X}}{\longrightarrow} \bar{G} \bar{F}(X) \stackrel{\lambda_{\bar{F} X}}{\longrightarrow} G \bar{F}(X) \stackrel{\gamma_{\bar{F} X}}{\longrightarrow} Q_{\bar{F} X}^{-}
$$

in $\mathscr{D}^{\mathrm{b}}(A)$. Applying $G$ to the first triangle, we obtain the following commutative diagram in $\mathscr{D}^{\mathrm{b}}(A)$ :

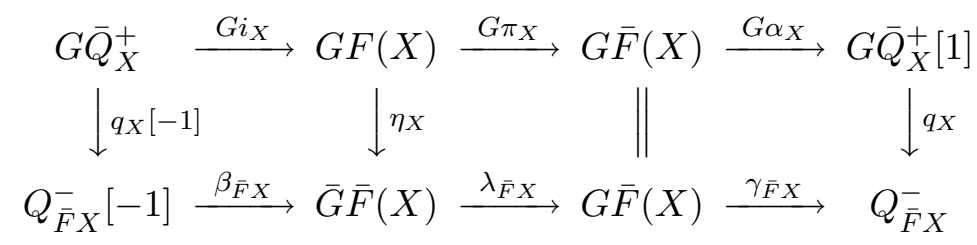

The existence of $\eta_{X}$ follows from the fact that $G\left(\pi_{X}\right) \gamma_{\bar{F} X}$ belongs to

$$
\begin{aligned}
\operatorname{Hom}_{\mathscr{D}^{\mathrm{b}}(A)}\left(G F(X), Q_{\overline{\bar{F} X}}^{-}\right) & \simeq \operatorname{Hom}_{\mathscr{D}^{\mathrm{b}}(A)}\left(X, Q_{\overline{\bar{F} X}}^{-}\right) \\
& \simeq \operatorname{Hom}_{\mathscr{K}^{\mathrm{b}}(A)}\left(X, Q_{\overline{\bar{F} X}}^{-}\right)=0 .
\end{aligned}
$$

Since $G F$ is naturally isomorphic to the identity functor $1_{\mathscr{D}}(A)$, there is a natural morphism $\epsilon_{X}: X \longrightarrow G F(X)$ in $\mathscr{D}^{\mathrm{b}}(A)$ for each $A$-module $X$. Let $\theta_{X}$ be the composition $\epsilon_{X} \eta_{X}$. Then $\theta_{X}: X \longrightarrow \bar{G} \bar{F}(X)$ is an $A$-module homomorphism since $A$-mod is fully embedded in $\mathscr{D}^{\mathrm{b}}(A)$.

We claim that $\theta_{X}$ is a natural map in $A$-mod. Indeed, for any $A$-module homomorphism $f: X \rightarrow Y$, by the proof of Proposition 3.4, we have a homomorphism $b_{f}: \bar{F}(X) \longrightarrow \bar{F}(Y)$ of $B$-modules such that $\pi_{X} b_{f}=F(f) \pi_{Y}$ in $\mathscr{D}^{\mathrm{b}}(B)$. By the proof of Proposition 3.5, there is a homomorphism $u_{b_{f}}$ : $\bar{G}(\bar{F}(X)) \longrightarrow \bar{G}(\bar{F}(Y))$ of $A$-modules such that $u_{b_{f}} \lambda_{\bar{F} Y}=\lambda_{\bar{F} X} G\left(b_{f}\right)$ in $\mathscr{D}^{\mathrm{b}}(A)$. Thus, we have in $\mathscr{D}^{\mathrm{b}}(A)$ :

$$
\begin{aligned}
\left(\theta_{X} u_{b_{f}}-f \theta_{Y}\right) \lambda_{\bar{F} Y} & =\left(\epsilon_{X} \eta_{X} u_{b_{f}}-f \epsilon_{Y} \eta_{Y}\right) \lambda_{\bar{F} Y} \\
& =\left(\epsilon_{X} \eta_{X} u_{b_{f}}-\epsilon_{X} G F(f) \eta_{Y}\right) \lambda_{\bar{F} Y} \\
& =\epsilon_{X}\left(\eta_{X} u_{b_{f}} \lambda_{\bar{F} Y}-G F(f) \eta_{Y} \lambda_{\bar{F} Y}\right)
\end{aligned}
$$




$$
\begin{aligned}
& =\epsilon_{X}\left(\eta_{X} \lambda_{\bar{F} X} G\left(b_{f}\right)-G F(f) \eta_{Y} \lambda_{\bar{F} Y}\right) \\
& =\epsilon_{X}\left(G\left(\pi_{X}\right) G\left(b_{f}\right)-G F(f) G\left(\pi_{Y}\right)\right) \\
& =\epsilon_{X}\left(G\left(\pi_{X}\right) G\left(b_{f}\right)-G\left(F(f) \pi_{Y}\right)\right) \\
& =\epsilon_{X}\left(G\left(\pi_{X}\right) G\left(b_{f}\right)-G\left(\pi_{X} b_{f}\right)\right) \\
& =0 .
\end{aligned}
$$

This implies that the map $\theta_{X} u_{b_{f}}-f \theta_{Y}$ factorizes through $Q_{\bar{F} Y}^{-}[-1]$. It follows by Lemma 3.3 that $\theta_{X} u_{b_{f}}-f \theta_{Y}$ factorizes through a projective module. Note that $u_{b_{f}}=\bar{G} \bar{F}(\underline{f})$. Thus, $\underline{\theta_{X}} \bar{G} \bar{F}(\underline{f})-\underline{f \theta_{Y}}=0$ in $A$ - $\underline{\bmod }$ and $\theta_{X}$ is natural in $X$.

To finish the proof, we have to show that $\theta_{X}$ is an isomorphism in $A$-mod for each $A$-module $X$. Clearly, we can assume that $X$ is an indecomposable nonprojective $A$-module. Using the method similar to that in the proof of Lemma 3.1, we can prove that $G\left(\bar{Q}_{X}^{+}\right)$is isomorphic in $\mathscr{D}^{\mathrm{b}}(A)$ to a radical complex $Q_{1}^{\bullet}$ in $\mathscr{K}^{\mathrm{b}}\left(A\right.$-proj) with $Q_{1}^{i} \in \operatorname{add}\left({ }_{A} Q\right)$ for all $i \leq 0$. Since both $X$ and $G \bar{F}(X)$ have zero homology in positive degrees, the complex $G\left(\bar{Q}_{X}^{+}\right)$ has zero homology in degrees greater than 1 , and therefore $Q_{1}^{i}=0$ for all $i>1$. Now we may form the following commutative diagram in $\mathscr{D}^{\mathrm{b}}(A)$ :

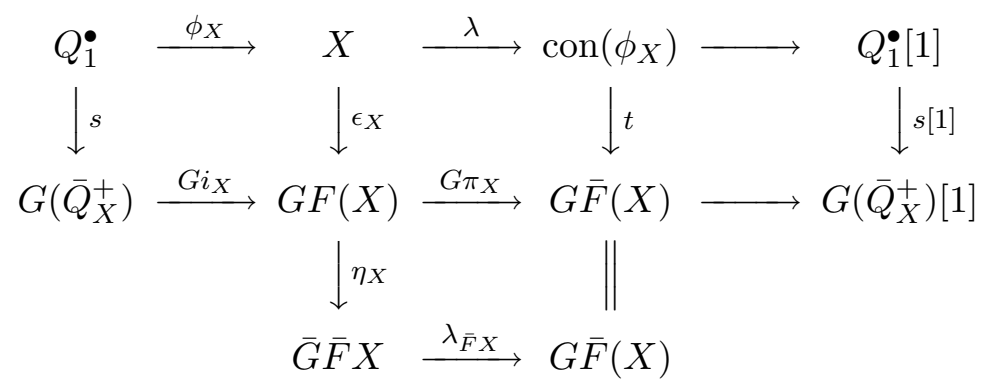

where $s$ is an isomorphism from $Q_{1}^{\bullet}$ to $G\left(Q_{X}^{+}\right), \phi_{X}=s G\left(i_{X}\right) \epsilon^{-1}$ is a chain map, and $\lambda$ is induced by the canonical map $\lambda^{0}$ from $X$ to $Q_{1}^{1} \oplus X$ defined by the mapping cone. Since $Q_{1}^{i}$ is in $\operatorname{add}\left({ }_{A} Q\right)$ for all $i \leq 0$ and zero for all $i>1$, the mapping cone $\operatorname{con}\left(\phi_{X}\right)$ has terms in $\operatorname{add}\left({ }_{A} Q\right)$ for all negative degrees and zero for all positive degrees. Note that $\operatorname{add}\left({ }_{A} Q\right)=\operatorname{add}\left(\nu_{A} Q\right)$ and that the $A$-module $Q$ is projective-injective. Consequently, the terms of $\operatorname{con}\left(\phi_{X}\right)$ in all negative degrees are projective-injective. Note that $G(\bar{F}(X))=Q_{\bar{F} X}^{\bullet}$ by our assumption. Thus, by definition (see Lemma 3.2), all terms of $Q_{\bar{F}}^{\bullet}$ in negative degrees are projective-injective. Moreover, both $\operatorname{con}\left(\phi_{X}\right)$ and 
$Q_{\bar{F} X}^{\bullet}$ have zero terms in all positive degrees. By Lemma 2.2(2), we have $\operatorname{Hom}_{\mathscr{D}^{\mathrm{b}}(A)}\left(\operatorname{con}\left(\phi_{X}\right), Q_{\bar{F} X}^{\bullet}\right)=\operatorname{Hom}_{\mathscr{K}^{\mathrm{b}}(A)}\left(\operatorname{con}\left(\phi_{X}\right), Q_{\bar{F} X}^{\bullet}\right)$. Since the two morphisms $\epsilon_{X}$ and $s$ are isomorphisms in $\mathscr{D}^{\mathrm{b}}(A)$, the morphism $t$ is also an isomorphism in $\mathscr{D}^{\mathrm{b}}(A)$. Hence, $t$ is an isomorphism from $\operatorname{con}\left(\phi_{X}\right)$ to $Q_{\bar{F} X}^{\bullet}$ in $\mathscr{K}^{\mathrm{b}}(A)$. Moreover, since $X$ is indecomposable and nonprojective, the complex $\operatorname{con}\left(\phi_{X}\right)$ is a radical complex. Thus, the chain map $t$ is actually an isomorphism between $\operatorname{con}\left(\phi_{X}\right)$ and $Q_{\bar{F} X}^{\bullet}$ in $\mathscr{C}^{b}(A)$, and the morphism $t^{0}:\left(\operatorname{con}\left(\phi_{X}\right)\right)^{0}=Q_{1}^{1} \oplus X \longrightarrow Q_{\bar{F} X}^{0}$ in degree 0 is an isomorphism of $A$-modules. From the above commutative diagram, we have $\theta_{X} \lambda_{\bar{F} X}-\lambda t=0$ in $\mathscr{D}^{\mathrm{b}}(A)$. By Lemma $2.2(2)$, we see that $\theta_{X} \lambda_{\bar{F} X}-\lambda t$ is null-homotopic in $\mathscr{C}^{b}(A)$. This means that $\theta_{X}-\lambda^{0} t^{0}$ factorizes through the projective $A$-module $Q_{\bar{F} X}^{-1}$. Hence, $\underline{\theta_{X}}=\underline{\lambda}^{0} \underline{t}^{0}$ is an isomorphism in $A$-mod since $\underline{\lambda}^{0}$ and $\underline{t}^{0}$ both are isomorphisms in $A$-mod.

REMARK. Without the condition $\operatorname{add}\left({ }_{A} Q\right)=\operatorname{add}\left(\nu_{A} Q\right)$ in Proposition 3.5, we can similarly define a functor $\bar{G}^{\prime}: B-\underline{\bmod } \longrightarrow A$ - $\underline{\bmod }$, as was done in Proposition 3.4. But the disadvantage of using $\bar{G}^{\prime}$ is that we do not know any behavior of the composition of $\bar{F}$ with $\bar{G}^{\prime}$.

We say that a derived equivalence $F$ between Artin algebras $A$ and $B$ is almost $\nu$-stable if $\operatorname{add}\left({ }_{A} Q\right)=\operatorname{add}\left(\nu_{A} Q\right)$ and $\operatorname{add}\left({ }_{B} \bar{Q}\right)=\operatorname{add}\left(\nu_{B} \bar{Q}\right)$.

The following theorem shows that the almost $\nu$-stable condition is sufficient for $\bar{F}$ to be an equivalence.

Theorem 3.7. Let $A$ and $B$ be two Artin R-algebras, and let $F$ : $\mathscr{D}^{\mathrm{b}}(A) \longrightarrow \mathscr{D}^{\mathrm{b}}(B)$ be a derived equivalence. If $F$ is almost $\nu$-stable, then the stable functor $\bar{F}$ is an equivalence.

Proof. Since $F$ is almost $\nu$-stable, we have $\operatorname{add}\left({ }_{A} Q\right)=\operatorname{add}\left(\nu_{A} Q\right)$. By

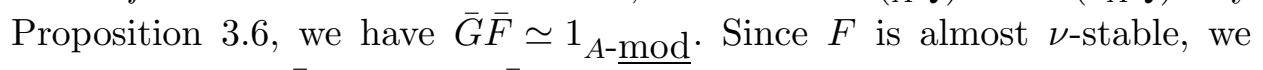
also have $\operatorname{add}\left({ }_{B} \bar{Q}\right)=\operatorname{add}\left(\nu_{B} \bar{Q}\right)$. With a proof similar to that of Proposition 3.6, we can show that $\bar{F} \bar{G}$ is naturally isomorphic to the identity functor $1_{B \text {-mod. }}$. Thus, $\bar{F}$ and $\bar{G}$ are equivalences of categories.

Theorem 3.7 gives rise to a method of getting stable equivalences from derived equivalences. In Section 5, we shall prove that, for finite-dimensional algebras, one can even get a stable equivalence of Morita type, which has many pleasant properties (see [3], [19], [20] and the references therein).

In the following, we develop some properties of almost $\nu$-stable functors, which will be used in Section 5 . 
Let ${ }_{A} E$ be a direct sum of all those nonisomorphic indecomposable projective-injective $A$-modules $X$ that have the property that $\nu_{A}^{i} X$ is again projective-injective for every $i>0$. The $A$-module ${ }_{A} E$ is unique up to isomorphism and is called the maximal $\nu$-stable $A$-module. Similarly, we have a maximal $\nu$-stable $B$-module ${ }_{B} \bar{E}$. Note that the maximal $\nu$-stable modules, and their endomorphism rings, have previously played an important role in the study of stable equivalences in [10].

The following result shows that an almost $\nu$-stable functor is closely related to the maximal $\nu$-stable modules.

Proposition 3.8. The following are equivalent:

(1) $F$ is almost $\nu$-stable; that is, $\operatorname{add}\left(\nu_{A} Q\right)=\operatorname{add}\left({ }_{A} Q\right)$ and $\operatorname{add}\left(\nu_{B} \bar{Q}\right)=$ $\operatorname{add}\left({ }_{B} \bar{Q}\right)$.

(2) ${ }_{A} Q \in \operatorname{add}\left({ }_{A} E\right)$, and ${ }_{B} \bar{Q} \in \operatorname{add}\left({ }_{B} \bar{E}\right)$.

(3) ${ }_{A} Q$ and $\nu_{B} \bar{Q}$ are projective-injective.

Proof. Clearly, we have $(1) \Rightarrow(2) \Rightarrow(3)$. Now we show that (3) implies (1). Assume that ${ }_{A} Q$ is injective. By Lemma 3.2, $G(B)$ is isomorphic in $\mathscr{D}^{\mathrm{b}}(A)$ to a radical complex $Q_{B}^{\bullet}=\left(Q_{B}^{i}, d^{i}\right)$ with $Q_{B}^{i}$ in $\operatorname{add}\left(\nu_{A} Q\right)$ for all $i<0$. In particular, $Q_{B}^{i}$ is injective for all $i<0$. Since $G(B) \simeq Q^{\bullet}$, the complexes $Q^{\bullet}$ and $Q_{B}^{\bullet}$ are isomorphic in $\mathscr{D}^{\mathrm{b}}(A)$. Since ${ }_{A} Q$ is injective by assumption, all the terms of $Q^{\bullet}$ in negative degrees are injective. By Lemma 2.2, the complexes $Q^{\bullet}$ and $Q_{B}^{\bullet}$ are isomorphic in $\mathscr{K}^{\mathrm{b}}(A)$. Since both $Q^{\bullet}$ and $Q_{B}^{\bullet}$ are radical, they are also isomorphic in $\mathscr{C}^{b}(A)$. In particular, we have $Q^{i} \simeq Q_{B}^{i}$ for all $i<0$, and therefore ${ }_{A} Q:=\bigoplus_{i=-1}^{-n} Q^{i} \simeq \bigoplus_{i=-1}^{-n} Q_{B}^{i} \in \operatorname{add}\left(\nu_{A} Q\right)$. Since ${ }_{A} Q$ and $\nu_{A} Q$ have the same number of isomorphism classes of indecomposable direct summands, we have $\operatorname{add}\left({ }_{A} Q\right)=\operatorname{add}\left(\nu_{A} Q\right)$. Similarly, we have $\operatorname{add}\left({ }_{B} \bar{Q}\right)=\operatorname{add}\left(\nu_{B} \bar{Q}\right)$. This proves $(3) \Rightarrow(1)$.

Remark. From Proposition 3.8, we can see that every derived equivalence between two self-injective Artin algebras induces an almost $\nu$-stable derived equivalence. Thus, we can reobtain the result of Rickard [13, Corollary 2.2] by Theorem 3.7.

Lemma 3.9. Suppose that $Q \in \operatorname{add}\left({ }_{A} E\right)$ and that $\bar{Q} \in \operatorname{add}\left({ }_{B} \bar{E}\right)$. Then,

(1) for each $P^{\bullet}$ in $\mathscr{K}^{\mathrm{b}}\left(\operatorname{add}\left({ }_{A} E\right)\right)$, the complex $F\left(P^{\bullet}\right)$ is isomorphic in $\mathscr{D}^{\mathrm{b}}(B)$ to a complex in $\mathscr{K}^{\mathrm{b}}\left(\operatorname{add}\left({ }_{B} \bar{E}\right)\right)$;

(2) for each $\bar{P}^{\bullet}$ in $\mathscr{K}^{\mathrm{b}}\left(\operatorname{add}\left({ }_{B} \bar{E}\right)\right)$, the complex $G\left(\bar{P}^{\bullet}\right)$ is isomorphic in $\mathscr{D}^{\mathrm{b}}(A)$ to a complex in $\mathscr{K}^{\mathrm{b}}\left(\operatorname{add}\left({ }_{A} E\right)\right)$. 
Proof. (1) It suffices to show that, for each indecomposable $A$-module $U$ in $\operatorname{add}\left({ }_{A} E\right)$, the complex $F(U)$ is isomorphic to a complex in $\mathscr{K}^{\mathrm{b}}\left(\operatorname{add}\left({ }_{B} \bar{E}\right)\right)$. Suppose that $U \in \operatorname{add}\left({ }_{A} E\right)$. By Lemma 3.1, $F(U)$ is isomorphic in $\mathscr{D}^{\mathrm{b}}(B)$ to a radical complex $\bar{Q}_{U}^{\bullet}$ :

$$
0 \longrightarrow \bar{Q}_{U}^{0} \longrightarrow \bar{Q}_{U}^{1} \longrightarrow \cdots \longrightarrow \bar{Q}_{U}^{n} \longrightarrow 0,
$$

with $\bar{Q}_{U}^{i} \in \operatorname{add}\left({ }_{B} \bar{Q}\right)$ for all $i>0$. For simplicity, we assume that $F(U)$ is just $\bar{Q}_{U}^{\bullet}$. Since $\bar{Q}_{U}^{i} \in \operatorname{add}\left({ }_{B} \bar{Q}\right) \subseteq \operatorname{add}\left({ }_{B} \bar{E}\right)$ for $i>0$, it remains to show that $\bar{Q}_{U}^{0}$ is in $\operatorname{add}\left({ }_{B} \bar{E}\right)$. Clearly, $\bar{Q}_{U}^{0}$ is projective since $U$ is projective. Note that we have an isomorphism $\nu_{B} F(U) \simeq F\left(\nu_{A} U\right)$ in $\mathscr{D}^{\mathrm{b}}(B)$, that is, that $\nu_{B} \bar{Q}_{U}^{\bullet}$ is isomorphic to $\bar{Q}_{\nu_{A} U}^{\bullet}$. Note that $\nu_{B} \bar{Q}_{U}^{i} \in \operatorname{add}\left(\nu_{B} \bar{Q}\right)$ for all $i>0$ and that $\operatorname{add}\left(\nu_{B} \bar{Q}\right) \subseteq \operatorname{add}\left({ }_{B} \bar{E}\right)$ by the definition of ${ }_{B} \bar{E}$. Thus, $\nu_{B} \bar{Q}_{U}^{i}$ is projective-injective for all $i>0$, and $\nu_{B} \bar{Q}_{U}^{\bullet}$ and $\bar{Q}_{\nu_{A} U}^{\bullet}$ are isomorphic in $\mathscr{K}^{\mathrm{b}}(B)$ by Lemma 2.2. Since both $\nu_{B} \bar{Q}_{U}^{\bullet}$ and $\bar{Q}_{\nu_{A} U}^{\bullet}$ are radical complexes, $\nu_{B} \bar{Q}_{U}^{\bullet}$ and $\bar{Q}_{\nu_{A} U}^{\bullet}$ are actually isomorphic in $\mathscr{C}^{b}(B)$, and particularly we have $\nu_{B} \bar{Q}_{U}^{0} \simeq \bar{Q}_{\nu_{A} U}^{0}$. Note that if $U \in \operatorname{add}\left({ }_{A} E\right)$, then $\nu_{A}^{i} U \in \operatorname{add}\left({ }_{A} E\right)$ for all $i \geq 0$ by definition. Hence, for each integer $m>0$, we have $\nu_{B}^{m}\left(\bar{Q}_{U}^{0}\right) \simeq$ $\nu_{B}^{m-1}\left(\bar{Q}_{\nu_{A} U}^{0}\right) \simeq \cdots \simeq \bar{Q}_{\nu_{A}^{m} U}^{0}$, and therefore $\nu_{B}^{m}\left(\bar{Q}_{U}^{0}\right)$ is projective-injective. Thus, $\bar{Q}_{U}^{0} \in \operatorname{add}\left({ }_{B} \bar{E}\right)$ by definition.

Statement (2) is a dual statement of (1).

The next result is a consequence of Lemma 3.9.

Corollary 3.10. If $F$ is an almost $\nu$-stable derived equivalence between Artin algebras $A$ and $B$, then there is a derived equivalence between the self-injective algebras $\operatorname{End}_{A}(E)$ and $\operatorname{End}_{B}(\bar{E})$.

Proof. By Lemma 3.9, $F$ induces an equivalence between $\mathscr{K}^{\mathrm{b}}\left(\operatorname{add}\left({ }_{A} E\right)\right)$ and $\mathscr{K}^{\mathrm{b}}\left(\operatorname{add}\left({ }_{B} \bar{E}\right)\right)$ as triangulated categories. Since $\mathscr{K}^{\mathrm{b}}\left(\operatorname{add}\left({ }_{A} E\right)\right)$ and $\mathscr{K}^{\mathrm{b}}\left(\operatorname{End}\left({ }_{A} E\right)\right.$-proj) are equivalent as triangulated categories, we obtain an equivalence between $\mathscr{K}^{\mathrm{b}}\left(\operatorname{End}\left({ }_{A} E\right)\right.$-proj $)$ and $\mathscr{K}^{\mathrm{b}}\left(\operatorname{End}\left({ }_{B} \bar{E}\right)-\right.$ proj$)$ as triangulated categories. By [14, Theorem 6.4], the algebras $\operatorname{End}_{A}(E)$ and $\operatorname{End}_{B}(\bar{E})$ are derived-equivalent. Note that $\operatorname{End}_{A}(E)$ is self-injective (see $[10])$.

Let us end this section by the following result, which tells us how to get an almost $\nu$-stable derived equivalence from a tilting module.

Let $A$ be an Artin algebra. Recall that an $A$-module $T$ is called a tilting module if 
(1) the projective dimension of $T$ is finite,

(2) $\operatorname{Ext}_{A}^{i}(T, T)=0$ for all $i>0$, and

(3) there is an exact sequence $0 \longrightarrow A \longrightarrow T^{0} \longrightarrow \cdots \longrightarrow T^{m} \longrightarrow 0$ of $A$-mod with each $T^{i}$ in $\operatorname{add}\left({ }_{A} T\right)$.

It is well known that a tilting $A$-module ${ }_{A} T$ induces a derived equivalence between $A$ and $\operatorname{End}_{A}(T)$ (see [5, Theorem 2.10, page 109] and [4, Theorem 2.1]).

Proposition 3.11. Let $A$ be an Artin algebra. Suppose that ${ }_{A} T$ is a tilting $A$-module with $B=\operatorname{End}_{A}(T)$. Let

$$
0 \longrightarrow P_{n} \stackrel{d_{n}}{\longrightarrow} P_{n-1} \longrightarrow \cdots \longrightarrow P_{0} \stackrel{d_{0}}{\longrightarrow} T \longrightarrow 0
$$

be a minimal projective resolution of ${ }_{A} T$. Set $P:=\bigoplus_{i=0}^{n-1} P_{i}$. If $\operatorname{add}\left({ }_{A} P\right)=$ $\operatorname{add}\left(\nu_{A} P\right)$, then there is an almost $\nu$-stable derived equivalence between $A$ and $B$.

Proof. By [4, Theorem 2.1], the functor $F^{\prime}={ }_{A} T \otimes_{B}^{\mathbf{L}}-: \mathscr{D}^{\mathrm{b}}(B) \longrightarrow \mathscr{D}^{\mathrm{b}}(A)$ is a derived equivalence. Now we denote $F^{\prime}[-n]$ by $F$. Let $P^{\bullet}$ be the complex

$$
0 \longrightarrow P_{n} \stackrel{d_{n}}{\longrightarrow} P_{n-1} \longrightarrow \cdots \longrightarrow P_{0} \longrightarrow 0
$$

with $P_{n}$ in degree 0 . Then we have $F(B)=\left({ }_{A} T \otimes_{B}^{\mathbf{L}} B\right)[-n]={ }_{A} T[-n] \simeq P^{\bullet}$ in $\mathscr{D}^{\mathrm{b}}(A)$. Let $G$ be a quasi-inverse of $F$. Then $G\left(P^{\bullet}\right) \simeq G(F(B)) \simeq B$ in $\mathscr{D}^{\mathrm{b}}(B)$, and therefore $P^{\bullet}$ is a radical tilting complex associated to $G$.

Since $\operatorname{add}\left(\nu_{A} P\right)=\operatorname{add}\left({ }_{A} P\right)$, the module ${ }_{A} P$ is projective-injective. Thus, ${ }_{A} P \in \operatorname{add}\left({ }_{A} T\right)$ and $P_{i} \in \operatorname{add}\left({ }_{A} T\right)$ for all $0 \leq i \leq n-1$. We denote by $T^{\bullet}$ the complex

$$
0 \longrightarrow P_{n-1} \oplus P \stackrel{\left[\begin{array}{c}
d_{n-1} \\
0
\end{array}\right]}{\longrightarrow} P_{n-2} \longrightarrow \cdots \longrightarrow P_{0} \stackrel{d_{0}}{\longrightarrow} T \longrightarrow 0
$$

with $T$ in degree 0 . Then $\operatorname{Hom}_{A}^{\bullet}\left(T, T^{\bullet}\right)$ is a complex in $\mathscr{K}^{\mathrm{b}}(B$-proj $)$, and

$$
\begin{aligned}
F\left(\operatorname{Hom}_{A}^{\bullet}\left(T, T^{\bullet}\right)\right) & ={ }_{A} T \otimes_{B}^{\mathbf{L}} \operatorname{Hom}_{A}^{\bullet}\left(T, T^{\bullet}\right)[-n] \simeq{ }_{A} T \otimes_{B}^{\bullet} \operatorname{Hom}_{A}^{\bullet}\left(T, T^{\bullet}\right)[-n] \\
& \simeq T^{\bullet}[-n] \simeq P_{n} \oplus P
\end{aligned}
$$

in $\mathscr{D}^{\mathrm{b}}(A)$. Since $P^{\bullet}$ is a tilting complex over $A$, we have ${ }_{A} A \in \operatorname{add}\left(P_{n} \oplus\right.$ $P)$. Thus, there is a radical complex $\bar{P}^{\bullet}$ in $\mathscr{K}^{\mathrm{b}}\left(B\right.$-proj) such that $\bar{P}^{\bullet} \in$ $\operatorname{add}\left(\operatorname{Hom}_{A}^{\bullet}\left(T, T^{\bullet}\right)\right)$ and $F\left(\bar{P}^{\bullet}\right) \simeq A$ in $\mathscr{D}^{\mathrm{b}}(A)$. By definition, $\bar{P}^{\bullet}$ is a tilting 
complex associated to $F$. (For the unexplained notations appearing in this proof, we refer the reader to Section 5 below.)

We claim that $G$ is almost $\nu$-stable. In fact, $\bigoplus_{i=-1}^{-n} \bar{P}^{i}$ is in $\operatorname{add}\left(\operatorname{Hom}_{A}(T\right.$, $P)$ ), and $\bigoplus_{i=1}^{n} P^{i}=\bigoplus_{i=0}^{n-1} P_{i}=P$. Let ${ }_{A} E$ (resp., ${ }_{B} \bar{E}$ ) be the maximal $\nu$-stable $A$-module (resp., $B$-module). Then ${ }_{A} P \in \operatorname{add}\left({ }_{A} E\right)$. Note that we have the following isomorphisms of $B$-modules:

$$
\begin{aligned}
\nu_{B} \operatorname{Hom}_{A}(T, P) & =D \operatorname{Hom}_{B}\left(\operatorname{Hom}_{A}(T, P), \operatorname{Hom}_{A}(T, T)\right) \\
& \simeq D \operatorname{Hom}_{A}(P, T) \simeq D\left(P^{*} \otimes_{A} T\right) \simeq \operatorname{Hom}_{A}\left(T, \nu_{A} P\right) .
\end{aligned}
$$

Since $\operatorname{add}\left({ }_{A} P\right)=\operatorname{add}\left(\nu_{A} P\right)$, we have

$$
\operatorname{add}\left(\nu_{B} \operatorname{Hom}_{A}(T, P)\right)=\operatorname{add}\left(\operatorname{Hom}_{A}(T, P)\right),
$$

that is, $\operatorname{Hom}_{A}(T, P) \in \operatorname{add}\left({ }_{B} \bar{E}\right)$. It follows that $\bigoplus_{i=-1}^{-n} \bar{P}^{i}$ is in $\operatorname{add}\left({ }_{B} \bar{E}\right)$. By Proposition 3.8, the functor $G$ is almost $\nu$-stable.

Remark. Let $A$ be a self-injective Artin algebra, and let $X$ be an $A$-module. By [6, Corollary 3.7], there is a derived equivalence between $\operatorname{End}_{A}(A \oplus$ $X)$ and $\operatorname{End}_{A}\left(A \oplus \Omega_{A}(X)\right)$, which is induced by the almost add $(A)$-split sequence $0 \rightarrow \Omega_{A}(X) \rightarrow P_{X} \rightarrow X \rightarrow 0$, where $P_{X}$ is a projective cover of $X$. By Proposition 3.11, it is easy to check that this derived equivalence is almost $\nu$-stable. Thus, the algebras $\operatorname{End}_{A}(A \oplus X)$ and $\operatorname{End}_{A}\left(A \oplus \Omega_{A}(X)\right)$ are stably equivalent by Theorem 3.7 .

\section{§4. Comparison of homological dimensions}

In this section, we shall deduce some basic homological properties of the functor $\bar{F}$ and compare homological dimensions of $A$ with that of $B$. Throughout this article, we keep the notation introduced in the previous sections.

Recall that the finitistic dimension of an Artin algebra $A$, denoted by fin. $\operatorname{dim}(A)$, is defined to be the supremum of the projective dimensions of finitely generated $A$-modules of finite projective dimension. The finitistic dimension conjecture states that fin. $\operatorname{dim}(A)$ should be finite for any Artin algebra $A$. Concerning the new advances on this conjecture, we refer the reader to $[21]$ and the references therein.

For an $A$-module $X$, we denote by $\operatorname{pd}\left({ }_{A} X\right)$ the projective dimension of $X$, and by $\operatorname{gl} \operatorname{dim}(A)$ the global dimension of $A$, which is by definition the supremum of the projective dimensions of all finitely generated $A$-modules. Clearly, if $\operatorname{gl} \cdot \operatorname{dim}(A)<\infty$, then $\operatorname{fin} \cdot \operatorname{dim}(A)=\operatorname{gl} \cdot \operatorname{dim}(A)$. 
Proposition 4.1. Let $\bar{F}$ be the stable functor of $F$ defined in Proposition 3.4. Then,

(1) for each exact sequence $0 \longrightarrow X \stackrel{f}{\longrightarrow} Y \stackrel{g}{\longrightarrow} Z \longrightarrow 0$ in $A$-mod, there is an exact sequence

$$
0 \longrightarrow \bar{F}(X) \stackrel{\left[a, f^{\prime}\right]}{\longrightarrow} P \oplus \bar{F}(Y) \stackrel{\left[\begin{array}{c}
b \\
g^{\prime}
\end{array}\right]}{\longrightarrow} \bar{F}(Z) \longrightarrow 0
$$

in $B$-mod, where $P \in \operatorname{add}\left({ }_{B} \bar{Q}\right), \bar{F}(\underline{f})=\underline{f}^{\prime}$, and $\bar{F}(\underline{g})=\underline{g}^{\prime}$;

(2) for each $A$-module $X$, we have a B-module isomorphism: $\bar{F}\left(\Omega_{A}(X)\right) \simeq$ $\Omega_{B}(\bar{F}(X)) \oplus P$, where $P$ is a projective $B$-module and $\Omega$ is the syzygy operator;

(3) for each A-module $X$, we have

$$
\operatorname{pd}\left({ }_{B} \bar{F}(X)\right) \leq \operatorname{pd}\left({ }_{A} X\right) \leq \operatorname{pd}\left({ }_{B} \bar{F}(X)\right)+n
$$

(4) if $\bar{F}$ is an equivalence, then $A$ and $B$ have the same finitistic and global dimensions.

Proof. For each $A$-module $X$, we may assume that $F(X)$ is the complex $\bar{Q}_{X}^{\bullet}$ defined in Lemma 3.1.

(1) From the exact sequence $0 \longrightarrow X \stackrel{f}{\longrightarrow} Y \stackrel{g}{\longrightarrow} Z \longrightarrow 0$ in $A$-mod, we have a distinguished triangle in $\mathscr{D}^{\mathrm{b}}(A)$ :

$$
X \stackrel{f}{\longrightarrow} Y \stackrel{g}{\longrightarrow} Z \stackrel{\epsilon}{\longrightarrow} X[1]
$$

Applying the functor $F$, we get a distinguished triangle

$$
\bar{Q}_{X}^{\bullet} \stackrel{F(f)}{\longrightarrow} \bar{Q}_{Y}^{\bullet} \stackrel{F(g)}{\longrightarrow} \bar{Q}_{Z}^{\bullet} \stackrel{F(\epsilon)}{\longrightarrow} \bar{Q}_{X}^{\bullet}[1]
$$

in $\mathscr{D}^{\mathrm{b}}(B)$. Moreover, by Lemma 2.2, the morphisms $F(f)$ and $F(g)$ are induced by chain maps $p^{\bullet}$ and $q^{\bullet}$, respectively. So, we may assume that $F(f)=p^{\bullet}$ and $F(g)=q^{\bullet}$. Let $\operatorname{con}\left(q^{\bullet}\right)$ be the mapping cone of the chain map $q^{\bullet}$. Then we have a commutative diagram in $\mathscr{D}^{\mathrm{b}}(B)$,

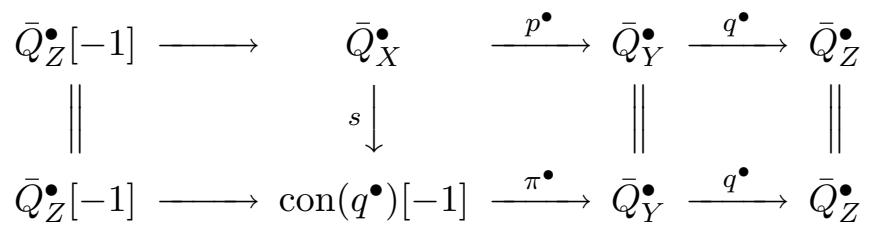


for some isomorphism $s$, where $\pi^{\bullet}=\left(\pi^{i}\right)$, with $\pi^{i}: \bar{Q}_{Y}^{i} \oplus \bar{Q}_{Z}^{i-1} \longrightarrow \bar{Q}_{Y}^{i}$ the canonical projection for each integer $i$. By Lemma 2.2, the morphism $s$ is induced by a chain map $s^{\bullet}$. By definition, $\operatorname{con}\left(s^{\bullet}\right)$ is the complex

$$
\begin{aligned}
0 & \longrightarrow \bar{Q}_{X}^{0} \stackrel{\left[-d, s^{0}\right]}{\longrightarrow} \bar{Q}_{X}^{1} \oplus \bar{Q}_{Y}^{0} \stackrel{\left[\begin{array}{ccc}
-d & u & v \\
0 & -d & q^{0}
\end{array}\right]}{\longrightarrow} \bar{Q}_{X}^{2} \oplus \bar{Q}_{Y}^{1} \oplus \bar{Q}_{Z}^{0} \\
& \longrightarrow \bar{Q}_{Z}^{n} \longrightarrow 0,
\end{aligned}
$$

where $s^{1}=[u, v]: \bar{Q}_{X}^{1} \longrightarrow \bar{Q}_{Y}^{1} \oplus \bar{Q}_{Z}^{0}$ and the modules $\bar{Q}_{X}^{i}, \bar{Q}_{Y}^{i}$, and $\bar{Q}_{Z}^{i}$ are projective for $i>0$. Since $s$ is an isomorphism in $\mathscr{D}^{\mathrm{b}}(B)$, the mapping cone $\operatorname{con}\left(s^{\bullet}\right)$ of $s^{\bullet}$ is an acyclic complex. Note that the map $-d: \bar{Q}_{Y}^{0} \longrightarrow \bar{Q}_{Y}^{1}$ is a radical map. Thus, dropping the split direct summands of the acyclic $\operatorname{complex} \operatorname{con}\left(s^{\bullet}\right)$, we get an exact sequence

$$
(*) \quad 0 \longrightarrow \bar{Q}_{X}^{0} \stackrel{\left[a, s^{0}\right]}{\longrightarrow} P \oplus \bar{Q}_{Y}^{0} \stackrel{\left[b, q^{0}\right]^{T}}{\longrightarrow} \bar{Q}_{Z}^{0} \longrightarrow 0
$$

in $B$-mod, where $P$ is a direct summand of $\bar{Q}_{X}^{1}$ and $a$ and $b$ are some homomorphisms of $B$-modules. It follows from $p^{\bullet}-s^{\bullet} \pi^{\bullet}=0$ that the morphism $p^{\bullet}-s^{\bullet} \pi^{\bullet}$ is null-homotopic by Lemma 2.2. Therefore, $p^{0}-s^{0}$ factorizes through $\bar{Q}_{X}^{1}$, and $p^{0}=\underline{s}^{0}$. By setting $f^{\prime}=s^{0}$ and $g^{\prime}=q^{0}$, we rewrite $(*)$ as

$$
0 \longrightarrow \bar{F}(X) \stackrel{\left[a, f^{\prime}\right]}{\longrightarrow} P \oplus \bar{F}(Y) \stackrel{\left[\begin{array}{c}
b \\
g^{\prime}
\end{array}\right]}{\longrightarrow} \bar{F}(Z) \longrightarrow 0
$$

with $P \in \operatorname{add}\left({ }_{B} \bar{Q}\right), \bar{F}(\underline{f})=\underline{f}^{\prime}$, and $\bar{F}(\underline{g})=\underline{g}^{\prime}$. This proves (1).

(2) Let $X$ be an $A$-module. We have an exact sequence $0 \longrightarrow \Omega_{A}(X) \longrightarrow$ $P_{X} \longrightarrow X \rightarrow 0$ in $A$-mod with $P_{X}$ projective. By (1), we get an exact sequence $0 \longrightarrow \bar{F}\left(\Omega_{A}(X)\right) \longrightarrow P \oplus \bar{F}\left(P_{X}\right) \longrightarrow \bar{F}(X) \longrightarrow 0$ in $B$-mod for some projective $B$-module $P$. By the definition of $\bar{F}$, the $B$-module $\bar{F}\left(P_{X}\right)$ is projective. Thus, (2) follows.

(3) The inequality $\operatorname{pd}\left({ }_{B} \bar{F}(X)\right) \leq \operatorname{pd}\left({ }_{A} X\right)$ follows from (2). In fact, we may assume that $\operatorname{pd}\left({ }_{A} X\right)=m<\infty$. Then $\Omega_{A}^{m}(X)$ is projective. Therefore, $\Omega_{B}^{m}(\bar{F}(X))$ is projective by $(2)$, and $\operatorname{pd}\left({ }_{B} \bar{F}(X)\right) \leq m$.

For the second inequality in (3), we may assume that $\operatorname{pd}\left({ }_{B} \bar{F}(X)\right)=m<$ $\infty$. Let $Y$ be an $A$-module. We claim that

$$
\operatorname{Ext}_{A}^{i}(X, Y) \simeq \operatorname{Hom}_{\mathscr{D} \mathrm{b}(A)}(X, Y[i]) \simeq \operatorname{Hom}_{\mathscr{D} \mathrm{b}(B)}(F(X), F(Y)[i])=0
$$

for all $i>m+n$. Indeed, by Lemma 3.1, the complex $F(X)$ is isomorphic in $\mathscr{D}^{\mathrm{b}}(B)$ to a complex $\bar{Q}_{X}^{\bullet}$ with $Q_{X}^{i}$ being projective for all $i>0$. Since 
$\operatorname{pd}\left({ }_{B} \bar{Q}_{X}^{0}\right)=\operatorname{pd}\left({ }_{B} \bar{F}(X)\right)=m$, we see that $\bar{Q}_{X}^{\bullet}$ is isomorphic in $\mathscr{D}^{\mathrm{b}}(B)$ to a complex $P^{\bullet}$ in $\mathscr{K}^{\mathrm{b}}\left(B\right.$-proj) with $P^{k}=0$ for all $k<-m$. Note that $F(Y)$ is isomorphic to the complex $\bar{Q}_{Y}^{\bullet}$ with $\bar{Q}_{Y}^{k}=0$ for all $k>n$. Clearly, we have

$$
\begin{aligned}
\operatorname{Hom}_{\mathscr{D}^{\mathrm{b}}(B)}(F(X), F(Y)[i]) & \simeq \operatorname{Hom}_{\mathscr{D}^{\mathrm{b}}(B)}\left(P^{\bullet}, \bar{Q}_{Y}^{\bullet}[i]\right) \\
& =\operatorname{Hom}_{\mathscr{K}^{\mathrm{b}}(B)}\left(P^{\bullet}, \bar{Q}_{Y}^{\bullet}[i]\right)=0
\end{aligned}
$$

for all $i>m+n$. Then the second inequality follows.

(4) This is a consequence of (2). In fact, suppose that $\bar{F}$ is an equivalence. Then, for an $A$-module $X$ and a positive integer $m$, the $A$-module $\Omega_{A}^{m}(X)$ is projective if and only if $\bar{F}\left(\Omega_{A}^{m}(X)\right)$ is projective. By $(2), \bar{F}\left(\Omega_{A}^{m}(X)\right)$ is projective if and only if $\Omega_{B}^{m}(\bar{F}(X))$ is projective. It follows that $\operatorname{pd}\left({ }_{A} X\right) \leq m$ if and only if $\operatorname{pd}\left({ }_{B} \bar{F}(X)\right) \leq m$, and consequently, $\operatorname{pd}\left({ }_{A} X\right)=\operatorname{pd}\left({ }_{B} \bar{F}(X)\right)$ for arbitrary $A$-module $X$. Thus, (4) follows.

REMARK. Proposition 4.1(3) can be regarded as an alternative proof of the result in [12] that if two Artin algebras $A$ and $B$ are derived-equivalent, then $\operatorname{fin} \cdot \operatorname{dim}(A)<\infty$ if and only if $\operatorname{fin} \cdot \operatorname{dim}(B)<\infty$.

In Proposition 3.5, we have constructed a functor $\bar{G}: B$ - $\underline{\bmod } \longrightarrow A$-mod under the condition $\operatorname{add}\left({ }_{A} Q\right)=\operatorname{add}\left(\nu_{A} Q\right)$. This functor $\bar{G}$ has many properties similar to those of $\bar{F}$.

Proposition 4.2. Suppose that $\operatorname{add}\left({ }_{A} Q\right)=\operatorname{add}\left(\nu_{A} Q\right)$. Let

$$
\bar{G}: B-\underline{\bmod } \longrightarrow A-\underline{\bmod }
$$

be the functor defined in Proposition 3.5. Then,

(1) for each exact sequence $0 \longrightarrow U \stackrel{f}{\longrightarrow} V \stackrel{g}{\longrightarrow} W \longrightarrow 0$ in $B$-mod, there is an exact sequence

$$
0 \longrightarrow \bar{G}(U) \stackrel{\left[a, f^{\prime}\right]}{\longrightarrow} P \oplus \bar{G}(V) \stackrel{\left[\begin{array}{c}
b \\
g^{\prime}
\end{array}\right]}{\longrightarrow} \bar{G}(W) \longrightarrow 0
$$

in $A$-mod, where $P \in \operatorname{add}\left({ }_{A} Q\right), \bar{G}(f)=f^{\prime}$, and $\bar{G}(g)=g^{\prime}$;

(2) for each $B$-module $Y$, we have $\bar{G}\left(\bar{\Omega}_{B}(Y)\right) \simeq \bar{\Omega}_{A}(\bar{G}(Y)) \oplus P$ in $A$-mod for a projective $A$-module $P$;

(3) for each $B$-module $Y$, we have $\operatorname{pd}\left({ }_{A} \bar{G}(Y)\right) \leq \operatorname{pd}\left({ }_{B} Y\right)$;

(4) if $I$ is an injective $B$-module, then $\bar{G}(I)$ is an injective A-module, and moreover, $\bar{G}(D(B)) \simeq \nu_{A} Q^{0}$. 
Proof. Statements (1), (2), and (3) are dual statements of Proposition 4.1, and their proofs will be omitted here. We prove only (4). Let $I$ be an injective $B$-module. Then $I=\nu_{B} P$ for a projective $B$-module $P$. Since $G(B) \simeq Q^{\bullet}$, we know that $G(P)$ is isomorphic in $\mathscr{K}^{\mathrm{b}}\left(A\right.$-proj) to a radical complex $Q_{1}^{\bullet} \in$ $\operatorname{add}\left(Q^{\bullet}\right)$. Thus, the complex $Q_{I}^{\bullet}$ defined in Lemma 3.2 is isomorphic to $G(I) \simeq \nu_{A} G(P) \simeq \nu_{A} Q_{1}^{\bullet}$ by Lemma 2.3. Moreover, all the terms of $\nu_{A} Q_{1}^{\bullet}$ in negative degrees are in $\operatorname{add}\left(\nu_{A} Q\right)$. Thus, by Lemma 3.2, the complexes $Q_{I}^{\bullet}$ and $\nu_{A} Q_{1}^{\bullet}$ are isomorphic in $\mathscr{C}^{b}(A)$, and consequently, $\bar{G}(I)=Q_{I}^{0} \simeq \nu_{A} Q_{1}^{0}$ is injective. In particular, if we take $P={ }_{B} B$ and $Q_{1}^{\bullet}=Q^{\bullet}$, then $\bar{G}(D(B))=$ $\bar{G}\left(\nu_{B} B\right) \simeq \nu_{A} Q^{0}$.

Let $A$ be an Artin algebra, and let $X$ be an $A$-module. Suppose that $0 \longrightarrow X \longrightarrow I_{0} \longrightarrow I_{1} \longrightarrow \cdots$ is a minimal injective resolution of $X$ with all $I_{j}$ injective. The dominant dimension of $X$, denoted by $\operatorname{dom} \cdot \operatorname{dim}(X)$, is defined to be

$$
\operatorname{dom} \operatorname{dim}(X):=\sup \left\{m \mid I_{i} \text { is projective for all } 0 \leq i \leq m-1\right\} \text {. }
$$

The dominant dimension of the algebra $A$, denoted by $\operatorname{dom} \cdot \operatorname{dim}(A)$, is defined to be the dominant dimension of the module ${ }_{A} A$. Concerning the dominant dimension of an Artin algebra, the Nakayama conjecture states that an Artin algebra with infinite dominant dimension should be selfinjective. It is well known that the finitistic dimension conjecture implies the Nakayama conjecture.

Usually, a derived equivalence does not preserve the global, finitistic, and dominant dimensions of an algebra. However, under the condition $\operatorname{add}\left({ }_{A} Q\right)=$ $\operatorname{add}\left(\nu_{A} Q\right)$, we have the following inequalities about these homological dimensions.

Corollary 4.3. Let $F: \mathscr{D}^{\mathrm{b}}(A) \longrightarrow \mathscr{D}^{\mathrm{b}}(B)$ be a derived equivalence between Artin algebras $A$ and $B$. If $\operatorname{add}\left({ }_{A} Q\right)=\operatorname{add}\left(\nu_{A} Q\right)$, then

(1) $\operatorname{fin} \cdot \operatorname{dim}(A) \leq$ fin. $\operatorname{dim}(B)$,

(2) $\operatorname{gl} \cdot \operatorname{dim}(A) \leq \operatorname{gl} \cdot \operatorname{dim}(B)$,

(3) $\operatorname{dom} \cdot \operatorname{dim}(A) \geq \operatorname{dom} \cdot \operatorname{dim}(B)$.

Proof.

(1) and (2) For each $A$-module $X$, we have $\operatorname{pd}\left({ }_{A} X\right)=\operatorname{pd}\left({ }_{A} \bar{G} \bar{F}(X)\right)$ by Proposition 3.6. According to Proposition 4.2(3), we have $\operatorname{pd}\left({ }_{A} \bar{G} \bar{F}(X)\right) \leq$ $\operatorname{pd}\left({ }_{B} \bar{F}(X)\right)$. By Proposition 4.1(3), we have another inequality $\operatorname{pd}\left({ }_{B} \bar{F}(X)\right) \leq$ $\operatorname{pd}\left({ }_{A} X\right)$. Thus, $\operatorname{pd}\left({ }_{A} X\right)=\operatorname{pd}\left({ }_{B} \bar{F}(X)\right)$. This implies that $\operatorname{gl} \cdot \operatorname{dim}(A) \leq$ 
gl.dim( $B)$. Moreover, if $\operatorname{pd}\left({ }_{A} X\right)<\infty$, we have $\operatorname{pd}\left({ }_{A} X\right)=\operatorname{pd}\left({ }_{B} \bar{F}(X)\right) \leq$ fin.dim $(B)$. Hence, fin.dim $(A) \leq f i n \cdot \operatorname{dim}(B)$. This proves $(1)$ and $(2)$.

(3) Suppose that $\operatorname{dom} \cdot \operatorname{dim}(B)=m$. Let $0 \longrightarrow{ }_{B} B \longrightarrow I_{0} \longrightarrow I_{1} \longrightarrow \cdots$ be a minimal injective resolution of ${ }_{B} B$. Then, by definition, the injective $B$-modules $I_{0}, \ldots, I_{m-1}$ are all projective. By Proposition 4.2(1), we get an exact sequence

$$
0 \longrightarrow{ }_{A} Q^{0} \longrightarrow P_{0} \oplus \bar{G}\left(I_{0}\right) \longrightarrow P_{1} \oplus \bar{G}\left(I_{1}\right) \longrightarrow \cdots
$$

with $P_{i} \in \operatorname{add}\left({ }_{A} Q\right)$. From this sequence we get another exact sequence

$$
0 \longrightarrow{ }_{A} Q^{0} \oplus{ }_{A} Q \longrightarrow P_{0} \oplus \bar{G}\left(I_{0}\right) \oplus{ }_{A} Q \longrightarrow P_{1} \oplus \bar{G}\left(I_{1}\right) \longrightarrow \cdots
$$

Since $\operatorname{add}\left({ }_{A} Q\right)=\operatorname{add}\left(\nu_{A} Q\right)$ and $\bar{G}\left(I_{i}\right)$ is injective for all $i$ by Proposition 4.2, the $A$-module $P_{i} \oplus \bar{G}\left(I_{i}\right)$ is injective for all $i$. Thus, the above exact sequence actually gives an injective resolution of ${ }_{A} Q^{0} \oplus_{A} Q$. Set $I_{0}^{\prime}:=P_{0} \oplus \bar{G}\left(I_{0}\right) \oplus{ }_{A} Q$, and set $I_{i}^{\prime}:=P_{i} \oplus \bar{G}\left(I_{i}\right)$ for $i>0$. Since $I_{i}$ is projective for all $i \leq m-1$, we see that $\bar{G}\left(I_{i}\right)$ is projective for all $0 \leq i \leq m-1$, and consequently, $I_{i}^{\prime}$ is projective for all $0 \leq i \leq m-1$. Hence, $\operatorname{dom} \cdot \operatorname{dim}\left({ }_{A} Q \oplus{ }_{A} Q^{0}\right) \geq m$. Moreover, since $Q^{\bullet}$ is a tilting complex, we have ${ }_{A} A \in \operatorname{add}\left(\bigoplus_{i} Q^{i}\right)$; that is, ${ }_{A} A \in \operatorname{add}\left({ }_{A} Q \oplus{ }_{A} Q^{0}\right)$. Hence, $\operatorname{dom} \cdot \operatorname{dim}(A) \geq m=\operatorname{dom} \cdot \operatorname{dim}(B)$.

\section{$\S 5$. Stable equivalences of Morita type induced by derived equiv- alences}

In this section, we shall prove that an almost $\nu$-stable derived equivalence $F$ between two finite-dimensional algebras actually induces a stable equivalence of Morita type. Our result in this section generalizes a well-known result of Rickard [15, Corollary 5.5], which states that, for finite-dimensional self-injective algebras, a derived equivalence induces a stable equivalence of Morita type.

Throughout this section, we keep the notations introduced in Section 3 and consider exclusively finite-dimensional algebras over a field $k$.

Let $\Lambda$ be an algebra. By $\mathscr{C}^{+}(\Lambda)$ (resp., $\mathscr{C}^{-}(\Lambda)$ ) we denote the full subcategory of $\mathscr{C}(\Lambda)$ consisting of all complexes bounded below (resp., bounded above). Analogously, one has the corresponding homotopy categories $\mathscr{K}^{+}(\Lambda)$ and $\mathscr{K}^{-}(\Lambda)$ as well as the corresponding derived categories $\mathscr{D}^{+}(\Lambda)$ and $\mathscr{D}^{-}(\Lambda)$. Recall that the category $\mathscr{D}^{-}(\Lambda)$ is equivalent to the category $\mathscr{K}^{-}(\Lambda$-proj $)$ and that the category $\mathscr{D}^{+}(\Lambda)$ is equivalent to the category 
$\mathscr{K}^{+}(\Lambda$-inj) (see, e.g., [18, Theorem 10.4.8, page 388]). Thus, for each complex $U^{\bullet}$ in $\mathscr{D}^{-}(\Lambda)$ (resp., $\mathscr{D}^{+}(\Lambda)$ ), we can find a complex $P_{U}^{\bullet} \in \mathscr{K}^{-}(\Lambda$-proj) (resp., $I_{U}^{\bullet} \in \mathscr{K}^{+}\left(\Lambda\right.$-inj)) that is isomorphic to $U^{\bullet}$ in $\mathscr{D}(\Lambda)$.

Now, let $X^{\bullet}$ be a complex in $\mathscr{D}^{-}\left(\Lambda^{\text {op }}\right)$, and let $Y^{\bullet}$ be a complex in $\mathscr{D}^{-}(\Lambda)$. By $X^{\bullet} \otimes_{\Lambda}^{\bullet} Y^{\bullet}$ we mean the total complex of the double complex with $(i, j)$ term $X^{i} \otimes_{\Lambda} Y^{j}$, and by $X^{\bullet} \otimes_{\Lambda}^{\mathbf{L}} Y^{\bullet}$ we mean the complex $X^{\bullet} \otimes_{\Lambda}^{\bullet} P_{Y}^{\bullet}$. Up to isomorphism of complexes in $\mathscr{D}(k)$, we know that $X^{\bullet} \otimes_{\Lambda}^{\mathbf{L}} Y^{\bullet}$ does not depend on the choice of $P_{Y}^{\bullet}$. It is known that $X^{\bullet} \otimes_{\Lambda}^{\mathbf{L}}-$ is a functor from $\mathscr{D}^{-}(\Lambda)$ to $\mathscr{D}^{-}(k)$ and is called the left derived functor of $X^{\bullet} \otimes_{\Lambda}-: \mathscr{K}^{-}(\Lambda) \longrightarrow \mathscr{K}^{-}(k)$. Note that if we choose $P_{X}^{\bullet} \in \mathscr{D}^{-}\left(\Lambda^{\text {op }}\right)$ such that $P_{X}^{\bullet} \simeq X^{\bullet}$ in $\mathscr{D}\left(\Lambda^{\text {op }}\right)$, then there is a natural isomorphism between $X^{\bullet} \otimes_{\Lambda}^{\bullet} P_{Y}^{\bullet}$ and $P_{X}^{\bullet} \otimes_{\Lambda}^{\bullet} Y^{\bullet}$ in $\mathscr{D}(k)$ (see [18, Exercise 10.6.1, page 395]). Thus, $X^{\bullet} \otimes_{\Lambda}^{\mathbf{L}} Y^{\bullet}$ can be calculated by $P_{X}^{\bullet} \otimes_{\Lambda}^{\bullet} Y^{\bullet}$. Let $X_{1}^{\bullet}$ be a complex in $\mathscr{D}^{-}\left(\Lambda^{\mathrm{op}}\right)$, and let $X_{2}^{\bullet}$ be a complex in $\mathscr{D}^{+}(\Lambda)$. By $\operatorname{Hom}_{\Lambda}^{\bullet}\left(X_{1}^{\bullet}, X_{2}^{\bullet}\right)$ we denote the total complex of the double complex with $(i, j)$-term $\operatorname{Hom}_{\Lambda}\left(X_{1}^{-i}, X_{2}^{j}\right)$. Choose $I_{X_{2}}^{\bullet} \in \mathscr{K}^{+}\left(\Lambda\right.$-inj) with $I_{X_{2}}^{\bullet} \simeq X_{2}^{\bullet}$ in $\mathscr{D}(\Lambda)$. We define $\mathbf{R H o m}_{\Lambda}\left(X_{1}^{\bullet}, X_{2}^{\bullet}\right)=\operatorname{Hom}_{\Lambda}^{\bullet}\left(X_{1}^{\bullet}, I_{X_{2}}^{\bullet}\right)$. It is known that $\mathbf{R H o m}_{\Lambda}\left(X_{1}^{\bullet},-\right): \mathscr{D}^{+}(\Lambda) \longrightarrow \mathscr{D}^{+}(k)$ is a functor. This functor is called the right derived functor of $\operatorname{Hom}_{\Lambda}\left(X_{1}^{\bullet},-\right): \mathscr{K}^{+}(\Lambda) \longrightarrow \mathscr{K}^{+}(k)$. Note that if we choose $P_{X_{1}}^{\bullet} \in \mathscr{K}^{-}\left(\Lambda\right.$-proj) with $P_{X_{1}}^{\bullet} \simeq X_{1}^{\bullet}$ in $\mathscr{D}(\Lambda)$, then the complexes $\operatorname{Hom}_{\Lambda}^{\bullet}\left(P_{X_{1}}^{\bullet}, X_{2}^{\bullet}\right)$ and $\operatorname{Hom}_{\Lambda}^{\bullet}\left(X_{1}^{\bullet}, I_{X_{2}}^{\bullet}\right)$ are naturally isomorphic in $\mathscr{D}(k)$ (see $[18$, Exercise 10.7 .1 , page 400$])$. Thus, $\mathbf{R H o m}_{\Lambda}\left(X_{1}^{\bullet}, X_{2}^{\bullet}\right)$ can be calculated by $\operatorname{Hom}_{\Lambda}^{\bullet}\left(P_{X_{1}}^{\bullet}, X_{2}^{\bullet}\right)$.

Suppose that $\Lambda_{1}$ and $\Lambda_{2}$ are two algebras. Let $T_{i}^{\bullet}$ be a tilting complex over $\Lambda_{i}$ with $\Gamma_{i}=\operatorname{End}_{\mathscr{D}}{ }_{\left(\Lambda_{i}\right)}\left(T_{i}^{\bullet}\right)$ for $i=1,2$. By a result of Rickard [15, Theorem 3.1], $T_{1}^{\bullet} \otimes_{k}^{\bullet} T_{2}^{\bullet}$ is a tilting complex over $\Lambda_{1} \otimes_{k} \Lambda_{2}$, and the endomorphism algebra of $T_{1}^{\bullet} \otimes_{k}^{\bullet} T_{2}^{\bullet}$ is canonically isomorphic to $\Gamma_{1} \otimes_{k} \Gamma_{2}$. Thus, the tensor products $\Lambda_{1} \otimes_{k} \Lambda_{2}$ and $\Gamma_{1} \otimes_{k} \Gamma_{2}$ are derived-equivalent.

Recall that $Q^{\bullet}$ is a tilting complex over $A$ with the endomorphism algebra $B$. By $[14$, Proposition 9.1$], \operatorname{Hom}_{A}^{\bullet}\left(Q^{\bullet}, A\right)$ is a tilting complex over $A^{\text {op }}$ with the endomorphism algebra $B^{\text {op }}$. Also, $\bar{Q}^{\bullet}$ is a tilting complex over $B$ with the endomorphism algebra $A$, and therefore $\operatorname{Hom}_{B}^{\bullet}\left(\bar{Q}^{\bullet}, B\right)$ is a tilting complex over $B^{\text {op }}$ with the endomorphism algebra $A$. Thus, by taking tensor products, we get four derived-equivalent algebras $A \otimes_{k} A^{\mathrm{op}}, A \otimes_{k} B^{\mathrm{op}}, B \otimes_{k}$ $B^{\text {op }}$, and $B \otimes_{k} A^{\text {op }}$. Table 1, taken from [15], describes the corresponding objects in various equivalent derived categories.

Using Table 1, one can easily find the corresponding objects in the above four equivalent derived categories. For instance, we consider the derived 
Table 1

\begin{tabular}{|c|c|c|c|}
\hline $\mathscr{D}^{\mathrm{b}}\left(A \otimes_{k} A^{\mathrm{op}}\right)$ & $\mathscr{D}^{\mathrm{b}}\left(A \otimes_{k} B^{\mathrm{op}}\right)$ & $\mathscr{D}^{\mathrm{b}}\left(B \otimes_{k} B^{\mathrm{op}}\right)$ & $\mathscr{D}^{\mathrm{b}}\left(B \otimes_{k} A^{\mathrm{op}}\right)$ \\
\hline$Q^{\bullet} \otimes_{k}^{\bullet} A_{A}$ & $Q^{\bullet} \otimes_{k}^{\bullet} \operatorname{Hom}_{B}^{\bullet}\left(\bar{Q}^{\bullet}, B\right)$ & ${ }_{B} B \otimes_{k}^{\bullet} \operatorname{Hom}_{B}^{\bullet}\left(\bar{Q}^{\bullet}, B\right)$ & ${ }_{B} B \otimes_{k} A_{A}$ \\
$Q^{\bullet} \otimes_{k}^{\bullet} \operatorname{Hom}_{A}\left(Q^{\bullet}, A\right)$ & $Q^{\bullet} \otimes_{k}^{\bullet} B_{B}$ & ${ }_{B} B \otimes_{k} B_{B}$ & ${ }_{B} B \otimes_{k}^{\bullet} \operatorname{Hom}_{A}^{\bullet}\left(Q^{\bullet}, A\right)$ \\
$A A \otimes_{k}^{\bullet} \operatorname{Hom}_{A}^{\bullet}\left(Q^{\bullet}, A\right)$ & $A A \otimes_{k} B_{B}$ & $\bar{Q}^{\bullet} \otimes_{k}^{\bullet} B_{B}$ & $\bar{Q}^{\bullet} \otimes_{k}^{\bullet} \operatorname{Hom}_{A}^{\bullet}\left(Q^{\bullet}, A\right)$ \\
${ }_{A} A \otimes_{k} A_{A}$ & $A A \otimes_{k}^{\bullet} \operatorname{Hom}_{B}^{\bullet}\left(\bar{Q}^{\bullet}, B\right)$ & $\bar{Q}^{\bullet} \otimes_{k}^{\bullet} \operatorname{Hom}_{B}\left(\bar{Q}^{\bullet}, B\right)$ & $\bar{Q}^{\bullet} \otimes_{k}^{\bullet} A_{A}$ \\
${ }_{A} A_{A}$ & $\Theta^{\bullet}$ & ${ }_{B} B_{B}$ & $\Delta^{\bullet}$ \\
\hline
\end{tabular}

equivalence $\widehat{F}: \mathscr{D}^{\mathrm{b}}\left(A \otimes_{k} A^{\mathrm{op}}\right) \longrightarrow \mathscr{D}^{\mathrm{b}}\left(B \otimes_{k} A^{\mathrm{op}}\right)$ induced by the tilting complex $Q^{\bullet} \otimes_{k}^{\bullet} A_{A}$. Table 1 shows that $\widehat{F}$ sends $Q^{\bullet} \otimes_{k}^{\bullet} A_{A}$ to $B B \otimes_{k} A_{A}, Q^{\bullet} \otimes_{k}^{\bullet}$ $\operatorname{Hom}_{A}^{\bullet}\left(Q^{\bullet}, A\right)$ to ${ }_{B} B \otimes_{k}^{\bullet} \operatorname{Hom}_{A}^{\bullet}\left(Q^{\bullet}, A\right),{ }_{A} A \otimes_{k}^{\bullet} \operatorname{Hom}_{A}^{\bullet}\left(Q^{\bullet}, A\right)$ to $\bar{Q}^{\bullet} \otimes_{k}^{\bullet} A_{A}$, and ${ }_{A} A_{A}$ to $\Delta^{\bullet}$. The following lemma collects some properties of these complexes (see [15]).

Lemma 5.1. Let $\Delta^{\bullet}$ and $\Theta^{\bullet}$ be the complexes defined in Table 1. We have the following.

(1) $\Delta^{\bullet} \otimes_{A}^{\mathbf{L}} \Theta^{\bullet} \simeq{ }_{B} B_{B}$ in $\mathscr{D}^{\mathrm{b}}\left(B \otimes_{k} B^{\mathrm{op}}\right)$.

(2) $\Theta^{\bullet} \otimes \otimes_{B}^{\mathrm{L}} \Delta^{\bullet} \simeq{ }_{A} A_{A}$ in $\mathscr{D}^{\mathrm{b}}\left(A \otimes_{k} A^{\mathrm{op}}\right)$.

(3) The functor $\Delta^{\bullet} \otimes_{A}^{\mathbf{L}}-: \mathscr{D}^{\mathrm{b}}(A) \longrightarrow \mathscr{D}^{\mathrm{b}}(B)$ is a derived equivalence with $\Delta^{\bullet} \otimes_{A}^{\mathbf{L}} X^{\bullet} \simeq F\left(X^{\bullet}\right)$ for all $X^{\bullet} \in \mathscr{D}^{\mathrm{b}}(A)$.

(4) The functor $\Theta^{\bullet} \otimes_{B}^{\mathbf{L}}-: \mathscr{D}^{\mathrm{b}}(B) \longrightarrow \mathscr{D}^{\mathrm{b}}(A)$ is a derived equivalence with $\Theta^{\bullet} \otimes_{B}^{\mathbf{L}} U^{\bullet} \simeq G\left(U^{\bullet}\right)$ for all $U^{\bullet} \in \mathscr{D}^{\mathrm{b}}(B)$.

(5) $\Theta^{\bullet} \simeq \mathbf{R H o m}_{B}\left(\Delta^{\bullet}, B\right)$ in $\mathscr{D}^{\mathrm{b}}\left(A \otimes_{k} B^{\mathrm{op}}\right)$.

(6) $\Delta^{\bullet}$ is isomorphic to $\bar{Q}^{\bullet}$ when considered as an object in $\mathscr{D}^{\mathrm{b}}(B)$ and to $\operatorname{Hom}_{A}\left(Q^{\bullet}, A\right)$ when considered as an object in $\mathscr{D}^{\mathrm{b}}\left(A^{\mathrm{op}}\right)$.

(7) $\Theta^{\bullet}$ is isomorphic to $Q^{\bullet}$ when considered as an object in $\mathscr{D}^{\mathrm{b}}(A)$ and to $\operatorname{Hom}_{B}\left(\bar{Q}^{\bullet}, B\right)$ when considered as an object in $\mathscr{D}^{\mathrm{b}}\left(B^{\mathrm{op}}\right)$.

Proof. Statements (1)-(5) follow from [15, Theorem 3.3, Proposition 4.1] and the remarks after [15, Definition 4.2]. Statements (6) and (7) are taken from [15, Proposition 3.1].

Note that it is an open question in [15] whether the two functors $F$ and $\Delta^{\bullet} \otimes_{A}^{\mathbf{L}}-$ are naturally isomorphic, although they have isomorphic images on each object by Lemma $5.1(3)$.

Recall that a complex $T^{\bullet}$ in $\mathscr{D}^{\mathrm{b}}\left(A \otimes_{k} B^{\mathrm{op}}\right)$ is called a two-sided tilting complex over $A \otimes_{k} B^{\text {op }}$ if there is a complex $\bar{T}^{\bullet}$ in $\mathscr{D}^{\mathrm{b}}\left(B \otimes_{k} A^{\mathrm{op}}\right)$ such that $T^{\bullet} \otimes_{B}^{\mathbf{L}} \bar{T}^{\bullet} \simeq{ }_{A} A_{A}$ in $\mathscr{D}^{\mathrm{b}}\left(A \otimes_{k} A^{\mathrm{op}}\right)$ and $\bar{T}^{\bullet} \otimes_{A}^{\mathbf{L}} T^{\bullet} \simeq{ }_{B} B_{B}$ in $\mathscr{D}^{\mathrm{b}}\left(B \otimes_{k} B^{\mathrm{op}}\right)$. 
In this case, the complex $\bar{T}^{\bullet}$ is called an inverse of $T^{\bullet}$. From Lemma 5.1, we see that $\Delta^{\bullet}$ and $\Theta^{\bullet}$ defined in Table 1 are mutually inverse two-sided tilting complexes over $A \otimes_{k} B^{\text {op }}$ and $B \otimes_{k} A^{\text {op }}$, respectively.

The following lemma, which is crucial in our later proofs, describes some properties of the terms of the two-sided tilting complex $\Delta^{\bullet}$ in Table 1 .

Lemma 5.2. The two-sided tilting complex $\Delta^{\bullet}$ is isomorphic in $\mathscr{D}^{\mathrm{b}}\left(B \otimes_{k}\right.$ $A^{\mathrm{op})}$ to a radical complex

$$
0 \longrightarrow \Delta^{0} \longrightarrow \Delta^{1} \longrightarrow \cdots \longrightarrow \Delta^{n} \longrightarrow 0
$$

with $\Delta^{i} \in \operatorname{add}\left({ }_{B} \bar{Q} \otimes_{k} Q_{A}^{*}\right)$ for all $i>0$.

Proof. Thanks to Table 1 , there is a derived equivalence $\widehat{F}: \mathscr{D}^{\mathrm{b}}\left(A \otimes_{k}\right.$ $\left.A^{\mathrm{op}}\right) \longrightarrow \mathscr{D}^{\mathrm{b}}\left(B \otimes_{k} A^{\mathrm{op}}\right)$. Moreover, the complexes $Q^{\bullet} \otimes_{k}^{\bullet} A_{A}$ and $\bar{Q}^{\bullet} \otimes_{k}^{\bullet} A_{A}$ are the associated tilting complexes to $\widehat{F}$ and its quasi-inverse, respectively. Note that the two complexes are radical and have the shape as assumed in Section 3. By Table 1, the two-sided tilting complex $\Delta^{\bullet}$ over $B \otimes_{k} A^{\text {op }}$ is isomorphic in $\mathscr{D}^{\mathrm{b}}\left(B \otimes_{k} A^{\text {op }}\right)$ to $\widehat{F}\left({ }_{A} A_{A}\right)$, and therefore, by Lemma 3.1 , the complex $\Delta^{\bullet}$ is isomorphic in $\mathscr{D}^{\mathrm{b}}\left(B \otimes_{k} A^{\text {op }}\right)$ to a radical complex $R^{\bullet}$ :

$$
0 \longrightarrow R^{0} \longrightarrow R^{1} \longrightarrow \cdots \longrightarrow R^{n} \longrightarrow 0
$$

with $R^{i} \in \operatorname{add}\left({ }_{B} \bar{Q} \otimes_{k} A_{A}\right)$ for all $i>0$.

Similarly, by Table 1 , there is a derived equivalence $\widetilde{F}: \mathscr{D}^{\mathrm{b}}\left(B \otimes_{k} B^{\mathrm{op}}\right) \longrightarrow$ $\mathscr{D}^{\mathrm{b}}\left(B \otimes_{k} A^{\text {op }}\right)$ induced by the tilting complex ${ }_{B} B \otimes_{k}^{\bullet} \operatorname{Hom}_{B}^{\bullet}\left(\bar{Q}^{\bullet}, B\right)$ over $B \otimes_{k} B^{\mathrm{op}}$. From Table 1 , we know that the complex ${ }_{B} B \otimes_{k}^{\bullet} \operatorname{Hom}_{A}^{\bullet}\left(Q^{\bullet}, A\right)$ is a tilting complex associated to the quasi-inverse of $\widetilde{F}$. Moreover, it follows from Table 1 that $\widetilde{F}\left({ }_{B} B_{B}\right) \simeq \Delta^{\bullet}$ in $\mathscr{D}^{\mathrm{b}}\left(B \otimes_{k} A^{\text {op }}\right)$. By Lemma $3.1, \widetilde{F}\left({ }_{B} B_{B}\right)$ is isomorphic in $\mathscr{D}^{\mathrm{b}}\left(B \otimes_{k} A^{\mathrm{op}}\right)$ to a radical complex $S^{\bullet}$ :

$$
0 \longrightarrow S^{0} \longrightarrow S^{1} \longrightarrow \cdots \longrightarrow S^{n} \longrightarrow 0
$$

with $S^{i} \in \operatorname{add}\left({ }_{B} B \otimes_{k} Q_{A}^{*}\right)$ for all $i>0$. Thus, both $R^{\bullet}$ and $S^{\bullet}$ are isomorphic to $\Delta^{\bullet}$ in $\mathscr{D}^{\mathrm{b}}\left(B \otimes_{k} A^{\text {op }}\right)$. By Lemma 2.2 , the complexes $R^{\bullet}$ and $S^{\bullet}$ are isomorphic in the homotopy category $\mathscr{K}^{\mathrm{b}}\left(B \otimes_{k} A^{\text {op }}\right)$. Since $R^{\bullet}$ and $S^{\bullet}$ are radical complexes, they are isomorphic in $\mathscr{C}^{b}\left(B \otimes_{k} A^{\mathrm{op}}\right)$. In particular, $R^{i} \simeq S^{i}$ as $B$-A-bimodules for all $i$. Thus, for each $i>0$, the bimodule $R^{i}$ lies in both $\operatorname{add}\left({ }_{B} B \otimes_{k} Q_{A}^{*}\right)$ and $\operatorname{add}\left({ }_{B} \bar{Q} \otimes_{k} A_{A}\right)$. As a result, we have $R^{i} \in \operatorname{add}\left({ }_{B} \bar{Q} \otimes_{k} Q_{A}^{*}\right)$ for all $i>0$. 
Using Lemma 5.2, we now prove the following main result in this section.

TheOREM 5.3. Let $A$ and $B$ be two finite-dimensional algebras over a field $k$, and let $F: \mathscr{D}^{\mathrm{b}}(A) \longrightarrow \mathscr{D}^{\mathrm{b}}(B)$ be a derived equivalence. If $F$ is almost $\nu$-stable, then there is a stable equivalence $\phi: A$-mod $\longrightarrow B$-mod of Morita type such that $\phi(X) \simeq \bar{F}(X)$ for any $A$-module $X$, where $\overline{\bar{F}}$ is defined in Proposition 3.4.

Proof. First, we show that $A$ may be assumed to be indecomposable. In fact, if $A=A_{1} \times A_{2}$ is a direct product of two algebras $A_{i}$, then the complex $Q^{\bullet}$ associated to $F$ has a decomposition $Q^{\bullet}=Q_{1}^{\bullet} \oplus Q_{2}^{\bullet}$ such that $Q_{1}^{\bullet} \in \mathscr{K}^{\mathrm{b}}\left(A_{1}\right.$-proj) and $Q_{2}^{\bullet} \in \mathscr{K}^{\mathrm{b}}\left(A_{2}\right.$-proj $)$. Correspondingly, the algebra $B$, which is isomorphic to the endomorphism algebra of $Q^{\bullet}$, is a product of two algebras, say, $B=B_{1} \times B_{2}$, such that $B_{i} \simeq \operatorname{End}_{\mathscr{D} \text { b }\left(A_{i}\right)}\left(Q_{i}^{\bullet}\right)$ for $i=1,2$. Thus, the derived equivalence $F: \mathscr{D}^{\mathrm{b}}(A) \longrightarrow \mathscr{D}^{\mathrm{b}}(B)$ induces two derived equivalences $F_{i}: \mathscr{D}^{\mathrm{b}}\left(A_{i}\right) \longrightarrow \mathscr{D}^{\mathrm{b}}\left(B_{i}\right)$ for $i=1,2$. Moreover, for each $i$, the complex $Q_{i}^{\bullet}$ is a tilting complex associated to $F_{i}$, and the tilting complex associated to the quasi-inverse of $F_{i}$ is isomorphic to $F_{i}\left(A_{i}\right) \simeq F\left(A_{i}\right)$, which is a direct summand of $\bar{Q}^{\bullet}$. Thus, if $F$ is almost $\nu$-stable, then $F_{i}$ is almost $\nu$-stable for $i=1,2$. Furthermore, if $A_{i}$ and $B_{i}$ are stably equivalent of Morita type for $i=1,2$, then $A_{1} \times A_{2}$ and $B_{1} \times B_{2}$ are stably equivalent of Morita type. Thus, we may assume that $A$ is indecomposable.

Since derived equivalence preserves the semisimplicity of algebras, we know that $A$ is semisimple if and only if $B$ is semisimple. Hence, we can further assume that $A$ is nonsemisimple. Now, let $A$ be nonsemisimple and indecomposable. Then $B$ is also nonsemisimple and indecomposable.

Let $\Delta^{\bullet}$ be the complex in Table 1 . By Lemma 5.2 , the complex $\Delta^{\bullet}$ is isomorphic in $\mathscr{D}^{\mathrm{b}}\left(B \otimes_{k} A^{\mathrm{op}}\right)$ to a radical complex

$$
0 \longrightarrow \Delta^{0} \longrightarrow \Delta^{1} \longrightarrow \cdots \longrightarrow \Delta^{n} \longrightarrow 0
$$

with $\Delta^{i}$ in $\operatorname{add}\left({ }_{B} \bar{Q} \otimes_{k} Q_{A}^{*}\right)$ for all $i>0$. For simplicity, we assume that $\Delta^{\bullet}$ is the above complex. By Lemma 5.1(6), the complex $\bar{Q}^{\bullet}$ is isomorphic to $\Delta^{\bullet}$ in $\mathscr{D}^{\mathrm{b}}(B)$. Thus, there is a chain map $\alpha: \bar{Q}^{\bullet} \rightarrow \Delta^{\bullet}$ such that the mapping cone $\operatorname{con}(\alpha)$ is acyclic. Since the terms of $\operatorname{con}(\alpha)$ in positive degrees are all projective, the acyclic complex $\operatorname{con}(\alpha)$ splits. This means that ${ }_{B} \Delta^{0}$ is a projective $B$-module. Thus, all terms of $\Delta^{\bullet}$ are projective as left $B$-modules. Similarly, by the fact that $\Delta^{\bullet}$ is isomorphic to $\operatorname{Hom}_{A}\left(Q^{\bullet}, A\right)$ in $\mathscr{D}^{\mathrm{b}}\left(A^{\mathrm{op}}\right)$, we infer that $\Delta_{A}^{0}$ is a projective right $A$-module and that all the terms of $\Delta^{\bullet}$ are 
projective as right $A$-modules. Consequently, the complex $\mathbf{R H o m}_{B}\left(\Delta^{\bullet}, B\right)$ is isomorphic in $\mathscr{D}^{\mathrm{b}}\left(A \otimes_{k} B^{\mathrm{op}}\right)$ to the complex $\operatorname{Hom}_{B}^{\bullet}\left(\Delta^{\bullet}, B\right)$ :

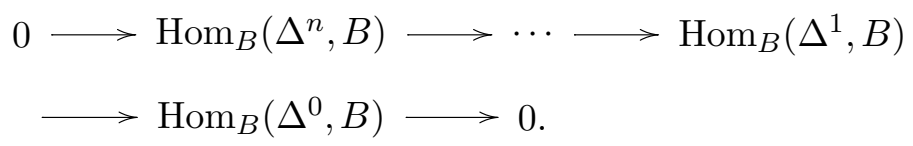

Since $\Delta^{i} \in \operatorname{add}\left({ }_{B} \bar{Q} \otimes_{k} Q_{A}^{*}\right)$ for all positive $i$, we find that the $A$-B-bimodule $\operatorname{Hom}_{B}\left(\Delta^{i}, B\right)$ is in $\operatorname{add}\left(\operatorname{Hom}_{B}\left({ }_{B} \bar{Q} \otimes_{k} Q_{A}^{*}, B\right)\right)$ for each positive integer $i$. Recall that there is a natural isomorphism

$$
\operatorname{Hom}_{B}\left({ }_{B} \bar{Q} \otimes_{k} Q_{A}^{*}, B\right) \simeq \nu_{A} Q \otimes_{k} \bar{Q}^{*} .
$$

Since $\operatorname{add}\left({ }_{A} Q\right)=\operatorname{add}\left(\nu_{A} Q\right)$, the term $\operatorname{Hom}_{B}\left(\Delta^{i}, B\right)$ is in $\operatorname{add}\left(Q \otimes \bar{Q}^{*}\right)$ for all $i>0$. As to $\operatorname{Hom}_{B}\left({ }_{B} \Delta_{A}^{0}, B\right)$, we can use Lemma 5.1(7) and similarly prove that $\operatorname{Hom}_{B}\left({ }_{B} \Delta_{A}^{0}, B\right)$ is projective as a one-sided module on both sides.

Next, we show that $\Delta^{0}$ and $\operatorname{Hom}_{B}\left(\Delta^{0}, B\right)$ define a stable equivalence of Morita type between $A$ and $B$. Indeed, by Lemma 5.1(5), the twosided tilting complex $\Theta^{\bullet}$ defined in Table 1 is isomorphic in $\mathscr{D}^{\mathrm{b}}\left(A \otimes_{k}\right.$ $\left.B^{\text {op }}\right)$ to $\mathbf{R H o m}_{B}\left(\Delta^{\bullet}, B\right)$, and the latter is isomorphic in $\mathscr{D}^{\mathrm{b}}\left(A \otimes_{k} B^{\mathrm{op}}\right)$ to $\operatorname{Hom}_{B}^{\bullet}\left(\Delta^{\bullet}, B\right)$. For simplicity, we assume that $\Theta^{\bullet}$ equals $\operatorname{Hom}_{B}^{\bullet}\left(\Delta^{\bullet}, B\right)$. Since all the terms of $\Delta^{\bullet}$ are projective as right $A$-modules, the complex $\Delta^{\bullet} \otimes_{A}^{\mathbf{L}} \Theta^{\bullet}$ is isomorphic in $\mathscr{D}^{\mathrm{b}}\left(B \otimes_{k} B^{\text {op }}\right)$ to the complex $\Delta^{\bullet} \otimes_{A}^{\bullet} \Theta^{\bullet}$. The $m$ th term of $\Delta^{\bullet} \otimes_{A}^{\bullet} \Theta^{\bullet}$ is

$$
\bigoplus_{i+j=m}\left(\Delta^{i} \otimes_{A} \Theta^{j}\right)=\bigoplus_{i+j=m}\left(\Delta^{i} \otimes_{A} \operatorname{Hom}_{B}\left(\Delta^{-j}, B\right)\right) .
$$

Let ${ }_{A} E$ be the maximal $\nu$-stable $A$-module, and let ${ }_{B} \bar{E}$ be the maximal $\nu$-stable $B$-module (see Section 3 ). Since $\operatorname{add}\left({ }_{A} Q\right)=\operatorname{add}\left(\nu_{A} Q\right)$ and $\operatorname{add}\left({ }_{B} \bar{Q}\right)=\operatorname{add}\left(\nu_{B} \bar{Q}\right)$, we have ${ }_{A} Q \in \operatorname{add}\left({ }_{A} E\right)$ and ${ }_{B} \bar{Q} \in \operatorname{add}\left({ }_{B} \bar{E}\right)$. By Lemmata 5.1(3) and 3.9, the complex $\Delta^{\bullet} \otimes_{A}^{\bullet} E$ is isomorphic in $\mathscr{D}^{\mathrm{b}}(B)$ to a complex in $\mathscr{K}^{\mathrm{b}}\left(\operatorname{add}\left({ }_{B} \bar{E}\right)\right)$. Since $\Delta^{i} \in \operatorname{add}\left({ }_{B} \bar{Q} \otimes_{k} Q_{A}\right)$ for $i>0$, the $i$ th term $\Delta^{i} \otimes_{A} E$ of $\Delta^{\bullet} \otimes_{A}^{\bullet} E$ is in $\operatorname{add}\left({ }_{B} \bar{Q}\right) \subseteq \operatorname{add}\left({ }_{B} \bar{E}\right)$ for all $i>0$. It follows that $\Delta^{0} \otimes_{A} E$ is in $\operatorname{add}\left({ }_{B} \bar{E}\right)$, and therefore

$$
E^{*} \otimes_{A} \Theta^{0}=E^{*} \otimes_{A} \operatorname{Hom}_{B}\left(\Delta^{0}, B\right) \simeq \operatorname{Hom}_{B}\left(\Delta^{0} \otimes_{A} E, B\right) \in \operatorname{add}\left(\bar{E}^{*}\right) .
$$

Note that, for each $i>0$, we have $\Delta^{i} \in \operatorname{add}\left(\bar{Q} \otimes_{k} Q^{*}\right) \subseteq \operatorname{add}\left(\bar{E} \otimes_{k} E^{*}\right)$ and $\Theta^{-i}=\operatorname{Hom}_{B}\left(\Delta^{i}, B\right) \in \operatorname{add}\left(Q \otimes_{k} \bar{Q}^{*}\right) \subseteq \operatorname{add}\left(E \otimes_{k} \bar{E}^{*}\right)$. Thus, it is not hard 
to see that $\Delta^{i} \otimes_{A} \Theta^{j}$ is in $\operatorname{add}\left(\bar{E} \otimes_{k} \bar{E}^{*}\right)$ for all $i$ and $j$ with $i+j \neq 0$. This means that all terms in nonzero degrees of $\Delta^{\bullet} \otimes_{A}^{\bullet} \Theta^{\bullet}$ are in $\operatorname{add}\left(\bar{E} \otimes_{k} \bar{E}^{*}\right)$. For the term in degree 0 of $\Delta^{\bullet} \otimes_{A}^{\bullet} \Theta^{\bullet}$, except $\Delta^{0} \otimes_{A} \Theta^{0}$, all of its other direct summands are in $\operatorname{add}\left(\bar{E} \otimes_{k} E^{*} \otimes_{A} E \otimes_{k} \bar{E}^{*}\right)$, which is contained in $\operatorname{add}\left(\bar{E} \otimes_{k} \bar{E}^{*}\right)$. Note that all the bimodules in $\operatorname{add}\left({ }_{B} \bar{E} \otimes_{k} \bar{E}_{B}^{*}\right)$ are projectiveinjective.

Now, we have $\Delta^{\bullet} \otimes_{A}^{\bullet} \Theta^{\bullet} \simeq{ }_{B} B_{B}$ in $\mathscr{D}^{\mathrm{b}}\left(B \otimes_{k} B^{\mathrm{op}}\right)$ by Lemma 5.1(1). Thus, the complex $\Delta^{\bullet} \otimes_{A}^{\bullet} \Theta^{\bullet}$ has zero homology and projective-injective terms in all nonzero degrees, and therefore it splits and is isomorphic to ${ }_{B} B_{B}$ in the homotopy category $\mathscr{K}^{\mathrm{b}}\left(B \otimes_{k} B^{\mathrm{op}}\right)$. Since $B$ is indecomposable and nonsemisimple, the bimodule ${ }_{B} B_{B}$ is indecomposable and nonprojective, and therefore it is a direct summand of $\Delta^{0} \otimes_{A} \Theta^{0}$. It follows that $\Delta^{0} \otimes_{A} \Theta^{0} \simeq$ ${ }_{B} B_{B} \oplus U$ for a projective-injective $B$ - $B$-bimodule $U$. Similarly, we have $\Theta^{0} \otimes_{B} \Delta^{0} \simeq{ }_{A} A_{A} \oplus V$ for a projective-injective $A$ - $A$-bimodule $V$. Hence, $A$ and $B$ are stably equivalent of Morita type.

Let $\phi: A$-mod $\longrightarrow B$ - $\underline{\bmod }$ be the stable equivalence induced by $\Delta^{0} \otimes_{A}-$. It follows from Lemmata 5.1(3) and 3.1 that $\phi(X) \simeq \bar{F}(X)$ in $B$ - $\underline{\bmod }$ for all $A$-modules $X$.

Let $A$ be an algebra. An $A$-module $M$ is called a generator-cogenerator for $A$-mod if $A \oplus D(A) \in \operatorname{add}(M)$. The representation dimension of $A$, denoted by $\operatorname{rep} \cdot \operatorname{dim}(A)$, is defined to be

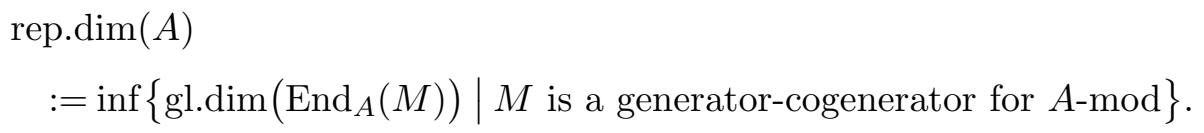

This notion was introduced by Auslander [1] to measure homologically how far an algebra is from being representation-finite and has been studied by many authors in recent years (see [17] and the references therein).

The following result is a consequence of Theorem 5.3 since stable equivalences of Morita type preserve representation dimensions (see [19]).

Corollary 5.4. If $F$ is an almost $\nu$-stable derived functor between $A$ and $B$, then $A$ and $B$ have the same representation and dominant dimensions.

As another consequence of Theorem 5.3, we reobtain the following result of Rickard [13] since every derived equivalence between self-injective algebras is, up to shift, almost $\nu$-stable by Proposition 3.8 . 
Corollary 5.5. Let $A$ and $B$ be finite-dimensional self-injective algebras. If $A$ and $B$ are derived-equivalent, then they are stably equivalent of Morita type.

Remark. (1) Let $A$ be a finite-dimensional self-injective algebra, and let $X$ be an $A$-module. By the remark at the end of Section 3, there is a derived equivalence between the algebras $\operatorname{End}_{A}(A \oplus X)$ and $\operatorname{End}_{A}(A \oplus$ $\Omega(X))$ satisfying the almost $\nu$-stable condition. Thus, we have an alternative proof of the result of Liu and Xi [9, Corollary 1.2] by applying Theorem 5.3.

(2) Theorem 5.3 may be false if only one of the two equalities of the almost $\nu$-stable condition is satisfied. For a counterexample, we refer the reader to Example 7.2 in Section 7.

\section{$\S 6$. Inductive constructions of almost $\nu$-stable derived equiva- lences}

In this section, we shall give several inductive constructions of almost $\nu$-stable derived equivalences. As a consequence, one can produce a lot of (usually not self-injective) finite-dimensional algebras that are both derivedequivalent and stably equivalent of Morita type.

In this section, we keep the notations introduced in Section 3. Our first inductive construction is the following proposition.

Proposition 6.1. Suppose that $F$ is an almost $\nu$-stable derived equivalence between finite-dimensional algebras $A$ and $B$ over a field $k$. Let $\bar{F}$ be the stable functor of $F$ defined in Proposition 3.4, and let $X$ be an A-module. Then there is an almost $\nu$-stable derived equivalence between the endomorphism algebras $\operatorname{End}_{A}(A \oplus X)$ and $\operatorname{End}_{B}(B \oplus \bar{F}(X))$.

Proof. We keep the notations in the proof of Theorem 5.3. By the last part of the proof of Theorem 5.3, the two-sided tilting complexes $\Delta^{\bullet}$ and $\Theta^{\bullet}$ have the properties $\Delta^{\bullet} \otimes_{A}^{\bullet} \Theta^{\bullet} \simeq{ }_{B} B_{B}$ in $\mathscr{K}^{\mathrm{b}}\left(B \otimes_{k} B^{\text {op }}\right)$ and $\Theta^{\bullet} \otimes_{B}^{\bullet} \Delta^{\bullet} \simeq{ }_{A} A_{A}$ in $\mathscr{K}^{\mathrm{b}}\left(A \otimes_{k} A^{\mathrm{op}}\right)$. It follows that the functor $\left(\Delta^{\bullet} \otimes_{A}^{\bullet} \Theta^{\bullet}\right) \otimes_{B}^{\bullet}-$ is naturally isomorphic to the identity functor $1_{\mathscr{K}^{\mathrm{b}}(B)}$ and that $\left(\Theta^{\bullet} \otimes_{B}^{\bullet} \Delta^{\bullet}\right) \otimes_{A}^{\bullet}$ - is naturally isomorphic to the identity functor $1_{\mathscr{K}^{\mathrm{b}}(A)}$. Thus, $\Delta^{\bullet} \otimes_{A}^{\bullet}-$ and $\Theta^{\bullet} \otimes_{B}^{\bullet}-$ induce mutually inverse equivalences between $\mathscr{K}^{\mathrm{b}}(A)$ and $\mathscr{K}^{\mathrm{b}}(B)$. Now we prove that the restrictions of these two functors to $\mathscr{K}^{\mathrm{b}}(\operatorname{add}(A \oplus X))$ and to $\mathscr{K}^{\mathrm{b}}(\operatorname{add}(B \oplus \bar{F}(X)))$ are also mutually inverse equivalences for each $A$-module $X$. 
In fact, the complex $\Delta^{\bullet} \otimes_{A}^{\bullet} X$ is of the form

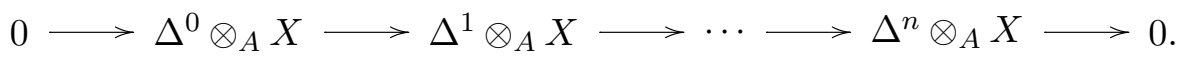

Since $\Delta^{i}$ is a projective bimodule for all $i>0$, the term $\Delta^{i} \otimes_{A} X$ is a projective $B$-module for all $i>0$. Moreover, by Theorem 5.3, we have $\Delta^{0} \otimes_{A} X \simeq \bar{F}(X)$ in $B$-mod, and therefore $\Delta^{0} \otimes_{A} X$ is a direct summand of $\bar{F}(X) \oplus P$ for some projective $B$-module $P$. Hence, the complex $\Delta^{\bullet} \otimes_{A}^{\bullet}$ $X$ is in $\mathscr{K}^{\mathrm{b}}(\operatorname{add}(B \oplus \bar{F}(X)))$. Note that, for each projective $A$-module $P_{1}$, the complex $\Delta^{\bullet} \otimes_{A}^{\bullet} P_{1}$ is in $\operatorname{add}\left(\bar{Q}^{\bullet}\right)$. Thus, for each complex $X^{\bullet}$ in $\mathscr{K}^{\mathrm{b}}(\operatorname{add}(A \oplus X))$, the complex $\Delta^{\bullet} \otimes_{A}^{\bullet} X^{\bullet}$ is in $\mathscr{K}^{\mathrm{b}}(\operatorname{add}(B \oplus \bar{F}(X)))$. Similarly, the functor $\Theta^{\bullet} \otimes_{B}^{\bullet}-$ takes complexes in $\mathscr{K}^{\mathrm{b}}(\operatorname{add}(B \oplus \bar{F}(X)))$ to complexes in $\mathscr{K}^{\mathrm{b}}(\operatorname{add}(A \oplus X))$. Thus, $\Delta^{\bullet} \otimes_{A}^{\bullet}-$ and $\Theta^{\bullet} \otimes_{B}^{\bullet}-$ induce mutually inverse equivalences between the triangulated categories $\mathscr{K}^{\mathrm{b}}(\operatorname{add}(A \oplus X))$ and $\mathscr{K}^{\mathrm{b}}(\operatorname{add}(B \oplus \bar{F}(X)))$.

Let $\Lambda=\operatorname{End}_{A}(A \oplus X)$, and let $\Gamma=\operatorname{End}_{B}(B \oplus \bar{F}(X))$. Then $\mathscr{K}^{\mathrm{b}}(\Lambda$-proj $)$ and $\mathscr{K}^{\mathrm{b}}(\Gamma$-proj $)$ are canonically equivalent to $\mathscr{K}^{\mathrm{b}}(\operatorname{add}(A \oplus X))$ and $\mathscr{K}^{\mathrm{b}}(\operatorname{add}(B \oplus \bar{F}(X)))$, respectively. By [14, Theorem 6.4], there is a derived equivalence $\widehat{F}$ between $\Lambda$ and $\Gamma$. Moreover, the tilting complexes associated to $\widehat{F}$ and its quasi-inverse are $\operatorname{Hom}_{A}^{\bullet}\left(A \oplus X, Q^{\bullet} \oplus \Theta^{\bullet} \otimes_{B}^{\bullet} \bar{F}(X)\right)$ and $\operatorname{Hom}_{B}^{\bullet}\left(B \oplus \bar{F}(X), \bar{Q}^{\bullet} \oplus \Delta^{\bullet} \otimes_{A}^{\bullet} X\right)$, respectively. By the proof of Theorem 5.3, the $i$ th term $\Theta^{i}$ of $\Theta^{\bullet}$ is in $\operatorname{add}\left(Q \otimes_{k} \bar{Q}^{*}\right)$ for all $i<0$. Hence, $\Theta^{i} \otimes_{B} \bar{F}(X)$ is in $\operatorname{add}(Q)$ for all $i<0$, and all the terms in negative degrees of $\operatorname{Hom}_{A}^{\bullet}\left(A \oplus X, Q^{\bullet} \oplus \Theta^{\bullet} \otimes_{B}^{\bullet} \bar{F}(X)\right)$ are in $\operatorname{add}\left(\operatorname{Hom}_{A}(A \oplus X, Q)\right)$. Similarly, all the terms in positive degrees of $\operatorname{Hom}_{B}^{\bullet}\left(B \oplus \bar{F}(X), \bar{Q}^{\bullet} \oplus \Delta^{\bullet} \otimes_{A}^{\bullet} X\right)$ are in $\operatorname{add}\left(\operatorname{Hom}_{B}(B \oplus \bar{F}(X), \bar{Q})\right)$. Note that we have the following isomorphisms:

$$
\begin{aligned}
\nu_{\Lambda}\left(\operatorname{Hom}_{A}(A \oplus X, Q)\right) & =D \operatorname{Hom}_{\Lambda}\left(\operatorname{Hom}_{A}(A \oplus X, Q), \operatorname{Hom}_{A}(A \oplus X, A \oplus X)\right) \\
& \simeq D \operatorname{Hom}_{A}(Q, A \oplus X) \\
& \simeq D\left(\operatorname{Hom}_{A}(Q, A) \otimes_{A}(A \oplus X)\right) \\
& \simeq \operatorname{Hom}_{A}\left(A \oplus X, D \operatorname{Hom}_{A}(Q, A)\right) \\
& =\operatorname{Hom}_{A}\left(A \oplus X, \nu_{A} Q\right) .
\end{aligned}
$$

Since $\operatorname{add}\left({ }_{A} Q\right)=\operatorname{add}\left(\nu_{A} Q\right)$, we have

$$
\operatorname{add}\left(\operatorname{Hom}_{A}(A \oplus X, Q)\right)=\operatorname{add}\left(\nu_{\Lambda}\left(\operatorname{Hom}_{A}(A \oplus X, Q)\right)\right) .
$$


Similarly, we have $\operatorname{add}\left(\operatorname{Hom}_{B}(B \oplus \bar{F}(X), \bar{Q})\right)=\operatorname{add}\left(\nu_{\Gamma}\left(\operatorname{Hom}_{B}(B \oplus \bar{F}(X)\right.\right.$, $\bar{Q}))$ ). This shows that the derived equivalence between $\Lambda$ and $\Gamma$ induced by the tilting complex $\operatorname{Hom}_{A}^{\bullet}\left(A \oplus X, Q^{\bullet} \oplus \Theta^{\bullet} \otimes_{B}^{\bullet} \bar{F}(X)\right)$ is almost $\nu$-stable.

Our next construction uses tensor products.

Proposition 6.2. Let $k$ be a field. Suppose that $F$ is an almost $\nu$-stable derived equivalence between finite-dimensional $k$-algebras $A$ and $B$. Then, for each finite-dimensional self-injective $k$-algebra $C$, there is an almost $\nu$ stable derived equivalence between the tensor products $A \otimes_{k} C^{\mathrm{op}}$ and $B \otimes_{k}$ $C^{\text {op }}$.

Proof. By [15, Theorem 2.1], $F$ induces a derived equivalence $\widehat{F}$ between $A \otimes_{k} C^{\text {op }}$ and $B \otimes_{k} C^{\text {op }}$. Let $Q^{\bullet}$ and $\bar{Q}^{\bullet}$ be the associated tilting complexes of $F$ and its quasi-inverse, respectively. From Table 1 we know that $Q^{\bullet} \otimes_{k}^{\bullet} C_{C}$ and $\bar{Q} \bullet \otimes_{k}^{\bullet} C_{C}$ are the associated tilting complexes of $\widehat{F}$ and its quasi-inverse, respectively. Now we have the following isomorphisms:

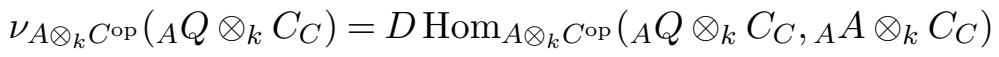

$$
\begin{aligned}
& \simeq D\left(\operatorname{Hom}_{A}\left({ }_{A} Q,{ }_{A} A\right) \otimes_{k} \operatorname{Hom}_{C} \text { op }\left(C_{C}, C_{C}\right)\right) \\
& \simeq D \operatorname{Hom}_{A}\left({ }_{A} Q,{ }_{A} A\right) \otimes_{k} D \operatorname{Hom}_{C} \text { op }\left(C_{C}, C_{C}\right) \\
& \simeq \nu_{A} Q \otimes_{k} \nu_{C^{\mathrm{op}}} C_{C} \\
& \simeq \nu_{A} Q \otimes_{k} C_{C} \quad \text { (because } C \text { is self-injective). }
\end{aligned}
$$

Since $F$ is almost $\nu$-stable, we have $\operatorname{add}(Q)=\operatorname{add}\left(\nu_{A} Q\right)$, and therefore

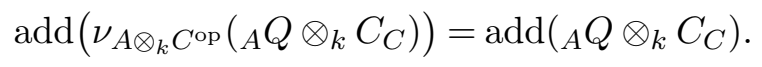

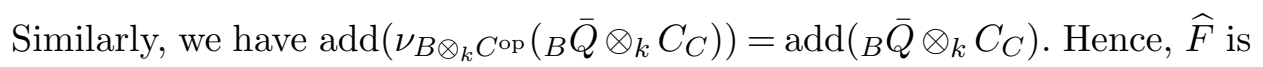
almost $\nu$-stable, and the proof is complete.

Let $A$ be a finite-dimensional algebra over a field $k$, and let $X$ be an $A$-module. The one-point extension of $A$ by $X$, denoted by $A[X]$, is the triangular matrix algebra $\left[\begin{array}{cc}k & 0 \\ X & A\end{array}\right]$. The canonical projection $A[X] \longrightarrow A$ shows that $A$ is a quotient algebra of $A[X]$ and that $A$-mod can be viewed as a full subcategory of $A[X]$-mod. Let $\widetilde{X}$ denote the $A[X]$-module $\left[\begin{array}{c}k \\ X\end{array}\right]$. Then, for each $A$-module $M$, we have $\operatorname{Hom}_{A[X]}(M, \widetilde{X}) \simeq \operatorname{Hom}_{A}(M, X)$.

Our third construction of an almost $\nu$-stable derived equivalence is given by one-point extensions. 
Proposition 6.3. Let $k$ be a field. Suppose that $F$ is an almost $\nu$-stable derived equivalence between finite-dimensional $k$-algebras $A$ and $B$. If $X$ is an $A$-module such that $F(X)$ is isomorphic in $\mathscr{D}^{\mathrm{b}}(B)$ to a B-module $Y$, then there is an almost $\nu$-stable derived equivalence between the one-point extensions $A[X]$ and $B[Y]$.

Proof. Let $G$ be a quasi-inverse of $F$. Recall that $Q^{\bullet}$ and $\bar{Q}^{\bullet}$ denote the radical tilting complexes associated to $F$ and $G$, respectively. Then $Q^{\bullet}$ can be viewed as a complex in $\mathscr{K}^{\mathrm{b}}(A[X]$-proj). By a result of Barot and Lenzing [2], the complex $Q^{\bullet} \oplus \tilde{X}$ is a tilting complex over $A[X]$ such that its endomorphism algebra is isomorphic to $B[Y]$, where $\tilde{X}$ is regarded as a complex concentrated only on degree 0 . Moreover, $\bar{Q}^{\bullet} \oplus \widetilde{Y}$ is a tilting complex associated to the quasi-inverse of the derived equivalence induced by $Q^{\bullet} \oplus \widetilde{X}$. Recall that $Q$ is the direct sum of all the terms of $Q^{\bullet}$ in negative degrees. Then $\operatorname{add}\left(\nu_{A} Q\right)=\operatorname{add}\left({ }_{A} Q\right)$ by assumption. Since the direct sum of all terms in negative degrees of $Q^{\bullet} \oplus \widetilde{X}$ equals $Q$, we have to show that $\operatorname{add}\left(\nu_{A[X]} Q\right)=\operatorname{add}\left({ }_{A[X]} Q\right)$.

Since $Q$ is projective, there is a unique maximal submodule $L$ of $X$ with respect to the property $\operatorname{Hom}_{A}(Q, L)=0$. By assumption, $F(X)$ is isomorphic in $\mathscr{D}^{\mathrm{b}}(B)$ to the $B$-module $Y$; hence, we have $\operatorname{Hom}\left(Q^{\bullet}, X[i]\right)=0$ for all $i \neq 0$. This shows that $0=\operatorname{Hom}_{\mathscr{D}^{\mathrm{b}}(A)}\left(Q^{\bullet}, X[i]\right) \simeq \operatorname{Hom}_{\mathscr{D}^{\mathrm{b}}(A)}\left(Q^{\bullet},(X / L)[i]\right)$ for all integers $i>0$. If $X / L \neq 0$, then $\operatorname{Hom}_{A}(Q, \operatorname{soc}(X / L)) \neq 0$ by the definition of $L$. This implies that $\operatorname{Hom}_{\mathscr{D}^{\mathrm{b}}(A)}\left(Q^{\bullet},(X / L)[i]\right) \neq 0$ for some $i>0$, a contradiction. Thus, $X / L=0, \operatorname{Hom}_{A}(Q, X)=0$, and $\operatorname{Hom}_{A[X]}(Q, \widetilde{X})=0$. Consequently,

$$
\begin{aligned}
\nu_{A[X]} Q & =D \operatorname{Hom}_{A[X]}(Q, A[X]) \simeq D \operatorname{Hom}_{A[X]}(Q, A \oplus \widetilde{X}) \\
& =D \operatorname{Hom}_{A[X]}(Q, A) \simeq \nu_{A} Q .
\end{aligned}
$$

Hence, $\operatorname{add}\left(\nu_{A[X]} Q\right)=\operatorname{add}\left({ }_{A[X]} Q\right)$. Similarly, we have $\operatorname{add}\left(\nu_{B[Y]} \bar{Q}\right)=$ $\operatorname{add}(B[Y] \bar{Q})$. This finishes the proof.

\section{$\S 7 . \quad$ Examples and questions}

In the following, we shall illustrate our results with examples.

ExAmple 1. Let $A$ and $B$ be finite-dimensional $k$ algebras given by quivers with relations in Figure 1 and Figure 2, respectively. Let $P_{A}(i)$, $I_{A}(i)$, and $S_{A}(i)$ denote the indecomposable projective, injective, and simple $A$-modules corresponding to the vertex $i$, respectively. We take a nonzero 


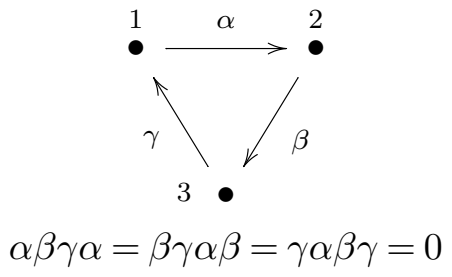

Figure 1

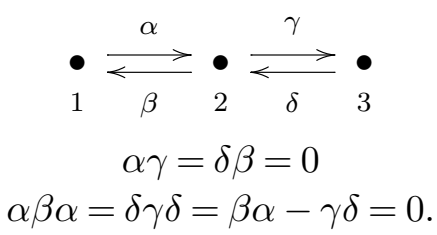

Figure 2

homomorphism $f: P_{A}(2) \rightarrow P_{A}(1)$. Then there is a tilting complex of $A$ modules

$$
Q^{\bullet}: 0 \longrightarrow P_{A}(2) \oplus P_{A}(2) \oplus P_{A}(3) \stackrel{[f, 0,0]^{T}}{\longrightarrow} P_{A}(1) \longrightarrow 0 .
$$

The endomorphism algebra of $Q^{\bullet}$ is isomorphic to $B$. Let $F: \mathscr{D}^{\mathrm{b}}(A) \rightarrow$ $\mathscr{D}^{\mathrm{b}}(B)$ be a derived equivalence with $Q^{\bullet}$ as its associated tilting complex. Clearly, $F$ is almost $\nu$-stable since $A$ and $B$ are symmetric algebras. Now we consider the quotient algebras $\bar{A}=A / \operatorname{soc}\left(P_{A}(1)\right)$ and $\bar{B}=B / \operatorname{soc}\left(P_{B}(1)\right)$. Then one can check that the complex

$$
Q_{1}^{\bullet}: 0 \longrightarrow P_{\bar{A}}(2) \oplus P_{\bar{A}}(2) \oplus P_{\bar{A}}(3) \stackrel{[\bar{f}, 0,0]^{T}}{\longrightarrow} P_{\bar{A}}(1) \longrightarrow 0
$$

is a tilting complex over $\bar{A}$ and that the endomorphism algebra of $Q_{1}^{\bullet}$ is isomorphic to $\bar{B}$. In other words, $Q_{1}^{\bullet}$ induces a derived equivalence $F_{1}$ : $\mathscr{D}^{\mathrm{b}}(\bar{A}) \longrightarrow \mathscr{D}^{\mathrm{b}}(\bar{B})$. This follows also from [7, Theorem 4.3]. Moreover, the tilting complex associated to a quasi-inverse of $F_{1}$ is of the following form:

$$
\bar{Q}_{1}^{\bullet}: 0 \longrightarrow P_{\bar{B}}(1) \longrightarrow P_{\bar{B}}(2) \oplus P_{\bar{B}}(2) \oplus P_{\bar{B}}(3) \longrightarrow 0 .
$$

Clearly, the two complexes satisfy the conditions $\operatorname{add}\left({ }_{\bar{A}} Q_{1}\right)=\operatorname{add}\left(\nu_{\bar{A}} Q_{1}\right)$ and $\operatorname{add}\left({ }_{\bar{B}} \bar{Q}_{1}\right)=\operatorname{add}\left(\nu_{\bar{B}} \bar{Q}_{1}\right)$. Hence, the algebras $\bar{A}$ and $\bar{B}$ are both derivedequivalent and stably equivalent of Morita type by Theorem 5.3. We know that $F_{1}\left(S_{\bar{A}}(1)\right)$ is isomorphic to the simple $\bar{B}$-module $S_{\bar{B}}(1)$.

The one-point extensions $\bar{A}\left[S_{\bar{A}}(1)\right]$ and $\bar{B}\left[S_{\bar{B}}(1)\right]$ are given in Figure 3 and Figure 4, respectively.

By Proposition 6.3, there is a derived equivalence between $\bar{A}\left[S_{\bar{A}}(1)\right]$ and $\bar{B}\left[S_{\bar{B}}(1)\right]$, which induces a stable equivalence of Morita type. 


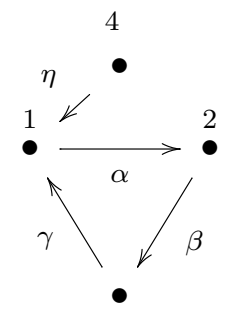

3

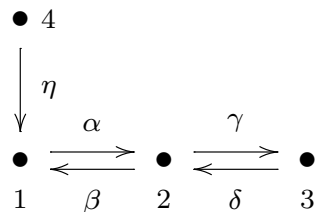

$\alpha \beta \gamma=\beta \gamma \alpha \beta=\gamma \alpha \beta \gamma=\eta \alpha=0$

Figure 3

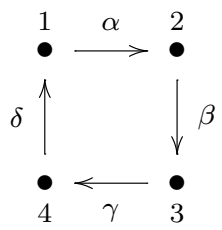

$\alpha \beta \gamma \delta=\beta \gamma \delta \alpha=\gamma \delta \alpha \beta \gamma=0$

Figure 5

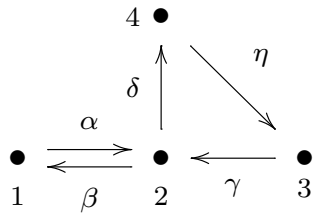

$\alpha \delta=\gamma \beta=\beta \alpha-\delta \eta \gamma=\gamma \delta \eta=\eta \gamma \delta=0$

Figure 6

A calculation shows that $F_{1}\left(I_{\bar{A}}(1)\right)$ is isomorphic to the $\bar{B}$-module $I_{\bar{B}}(1)$. The algebras $\operatorname{End}_{\bar{A}}\left(\bar{A} \oplus I_{\bar{A}}(1)\right)$ and $\operatorname{End}_{\bar{B}}\left(\bar{B} \oplus I_{\bar{B}}(1)\right)$ are given by Figures 5 and 6 , respectively. Thus, they are derived-equivalent and stably equivalent of Morita type by Proposition 6.1.

The next example, taken from [13], shows that Theorem 5.3 may fail if only one of the conditions of an almost $\nu$-stable functor is satisfied.

ExAmple 2. Let $A$ be the 17-dimensional algebra given by the quiver

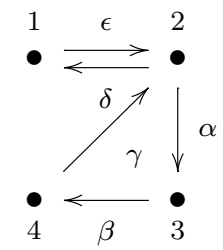

with relations $\gamma \alpha \beta=\gamma \delta=\epsilon \alpha \beta=0, \delta \epsilon=\alpha \beta \gamma$. As before, we denote by $P_{A}(i)$ the indecomposable projective $A$-module corresponding to the vertex $i$. Let 
$Q^{\bullet}$ be the direct sum of the following two complexes:

$$
\begin{gathered}
0 \longrightarrow P_{A}(1) \longrightarrow \begin{array}{c}
P_{A}(2) \\
0 \longrightarrow 0
\end{array} \quad \longrightarrow P_{A}(2) \oplus P_{A}(3) \oplus P_{A}(4) \longrightarrow 0 \\
0
\end{gathered}
$$

where $P_{A}(1)$ is in degree -1 . One can check that $Q^{\bullet}$ is a tilting complex over $A$. Let $B=\operatorname{End}_{\mathscr{D}^{\mathrm{b}}(A)}\left(Q^{\bullet}\right)$. Then $B$ is a 20-dimensional algebra given by the quiver

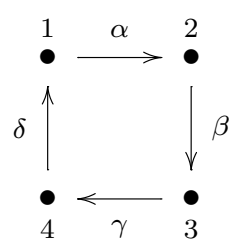

with relations $\alpha \beta \gamma \delta \alpha=0=\delta \alpha \beta \gamma$, where the indecomposable projective $B$-modules at vertices $1,2,3$, and 4 correspond respectively to the indecomposable direct summands $P_{A}(1) \rightarrow P_{A}(2), P_{A}(2), P_{A}(3)$, and $P_{A}(4)$ of the complex $Q^{\bullet}$. Let $F: \mathscr{D}^{\mathrm{b}}(A) \longrightarrow \mathscr{D}^{\mathrm{b}}(B)$ be the derived equivalence induced by the tilting complex $Q^{\bullet}$. Then $F\left(P_{A}(i)\right)=P_{B}(i)$ for $i=2,3,4$. Let $Q_{1}^{\bullet}$ be the direct summand $P_{A}(1) \rightarrow P_{A}(2)$ of $Q^{\bullet}$. Applying $F$ to the distinguished triangle in $\mathscr{D}^{\mathrm{b}}(A)$

$$
P_{A}(2)[-1] \longrightarrow Q_{1}^{\bullet}[-1] \longrightarrow P_{A}(1) \longrightarrow P_{A}(2),
$$

we see that $F\left(P_{A}(1)\right)$ is of the form

$$
0 \longrightarrow P_{B}(2) \longrightarrow P_{B}(1) \longrightarrow 0,
$$

where $P_{B}(2)$ is in degree 0 . Thus, $F(A)$ is isomorphic in $\mathscr{D}^{\mathrm{b}}(B)$ to a complex $\bar{Q}^{\bullet}$, which is the direct sum of the following two complexes:

$$
\begin{gathered}
0 \longrightarrow P_{B}(2) \\
0 \longrightarrow P_{B}(2) \oplus P_{B}(3) \oplus P_{B}(4) \longrightarrow P_{B}(1) \longrightarrow 0 \\
\longrightarrow 0
\end{gathered} \quad \text { ․ }
$$

Let $G$ be a quasi-inverse of $F$. Then $\bar{Q}^{\bullet}$ is a tilting complex associated to $G$. Clearly, ${ }_{A} Q=Q^{-1}=P_{A}(1)$ and ${ }_{B} \bar{Q}=\bar{Q}^{1}=P_{B}(1)$. It is easy to see that $F$ satisfies the condition $\operatorname{add}\left({ }_{B} \bar{Q}\right)=\operatorname{add}\left(\nu_{B} \bar{Q}\right)$ but not the condition $\operatorname{add}\left({ }_{A} Q\right)=\operatorname{add}\left(\nu_{A} Q\right)$. Note that $B$ is a Nakayama algebra and has 16 nonprojective indecomposable modules, while $A$ has more than 16 nonprojective indecomposable modules. Thus, $A$ and $B$ cannot be stably equivalent.

This example also shows that Corollary 3.10 may be false for derived equivalences in general. In fact, we have ${ }_{B} \bar{E}=P_{B}(1)$ and ${ }_{A} E=0$ in this example. 
Finally, we mention the following questions suggested by the results in this paper.

(1) What new conditions for a derived equivalence induce a stable equivalence of Morita type?

(2) Suppose that $R$ is an arbitrary commutative Artin ring. Does Theorem 5.3 hold true for Artin $R$-algebras $A$ and $B$ such that $A$ and $B$ both are projective over $R$ ?

Note that if $A$ and $B$ both are projective over $R$, then a two-sided tilting complex over $A \otimes_{R} B$ exists. However, we do not know whether $X \otimes_{R} Y$ is an injective $A \otimes_{R} B$-module if $X$ and $Y$ are injective over $A$ and $B$, respectively.

(3) Let $F: \mathscr{D}^{\mathrm{b}}(A) \longrightarrow \mathscr{D}^{\mathrm{b}}(B)$ be a derived equivalence between Artin algebras $A$ and $B$, and let $Q^{\bullet}$ be the tilting complex associated to $F$. If $\operatorname{add}\left({ }_{A} Q\right)=\operatorname{add}\left(\nu_{A} Q\right)$, is it true that $\operatorname{rep} \cdot \operatorname{dim}(A) \leq \operatorname{rep} \cdot \operatorname{dim}(B) ?$

\section{REFERENCES}

[1] M. Auslander, Representation Dimension of Artin Algebras, Queen Mary Coll. Math. Notes, Queen Mary College, London, 1971.

[2] M. Barot and H. Lenzing, One-point extensions and derived equivalences, J. Algebra 264 (2003), 1-5.

[3] M. Broué, "Equivalences of blocks of group algebras" in Finite Dimensional Algebras and Related Topics, Kluwer, Dordrecht, 1994, 1-26.

[4] E. Cline, B. Parshall, and L. Scott, Derived categories and Morita theory, J. Algebra 104 (1986), 397-409.

[5] D. Happel, Triangulated Categories in the Representation Theory of Finite Dimensional Algebras, Cambridge Univ. Press, Cambridge, 1988.

[6] W. $\mathrm{Hu}$ and C. C. Xi, Almost D-split sequences and derived equivalences, preprint, 2007, arXiv:0810.4757v1[math.RT]

[7] —, Derived equivalences for $\Phi$-Auslander-Yoneda algebras, preprint, 2009, arXiv:9012.0647v2[math.RT]

[8] H. Krause, Stable equivalence preserves representation type, Comment. Math. Helv. 72 (1997), 266-284.

[9] Y. M. Liu and C. C. Xi, Constructions of stable equivalences of Morita type for finite dimensional algebras, III, J. Lond. Math. Soc. (2) 76 (2007), 567-585.

[10] R. Martinez-Villa, Properties that are left invariant under stable equivalence, Comm. Algebra 18 (1990), 4141-4169.

[11] A. Neeman, Triangulated Categories, Princeton University Press, Princeton, 2001.

[12] S. Y. Pan and C. C. Xi, Finiteness of finitistic dimension is invariant under derived equivalences, J. Algebra 322 (2009), 21-24.

[13] J. Rickard, Derived categories and stable equivalences, J. Pure Appl. Algebra 64 (1989), 303-317.

[14] - Morita theory for derived categories, J. Lond. Math. Soc. (2) 39 (1989), $436-456$. 
[15] - Derived equivalence as derived functors, J. Lond. Math. Soc. (2) 43 (1991), $37-48$.

[16] - "The abelian defect group conjecture" in Proceedings of the International Congress of Mathematicians, Vol. II (Berlin, 1998), Doc. Math. 1998, Extra Vol. II, Documenta Mathematica, Bielefeld, 1998, 121-128.

[17] R. Rouquier, Representation dimension of exterior algebras, Invent. Math. 165 (2006), 357-367.

[18] C. A. Weibel, An Introduction to Homological Algebra, Cambridge University Press, Cambridge, 1994.

[19] C. C. Xi, Representation dimension and quasi-hereditary algebras, Adv. Math. 168 (2002), 193-212.

[20] - Stable equivalences of adjoint type, Forum Math. 20 (2008), 81-97.

[21] C. C. Xi and D. M. Xu, The finitistic dimension conjecture and relatively projective modules, preprint, 2007.

Wei $\mathrm{Hu}$

School of Mathematical Sciences

Laboratory of Mathematics and Complex Systems

Beijing Normal University

100875 Beijing

People's Republic of China

huwei@bnu.edu.cn

Changchang Xi (corresponding author)

School of Mathematical Sciences

Laboratory of Mathematics and Complex Systems

Beijing Normal University

100875 Beijing

People's Republic of China

xicc@bnu.edu.cn 